\title{
Efficient and Reliable Nonlocal Damage Models *
}

\author{
A. Rodríguez-Ferran *, I. Morata and A. Huerta \\ Laboratori de Càlcul Numèric (LaCàN), Universitat Politècnica de Catalunya, \\ Barcelona, Spain.
}

\begin{abstract}
We present an efficient and reliable approach for the numerical modelling of failure with nonlocal damage models. The two major numerical challenges - the strongly nonlinear, highly localized and parameter-dependent structural response of quasibrittle materials, and the interaction between non-adjacent finite elements associated to nonlocality - are addressed in detail. Reliability of the numerical results is ensured by an $h$-adaptive strategy based on error estimation. We use a residualtype error estimator for nonlinear FE analysis based on local computations, which, at the same time, accounts for the nonlocality of the damage model. Efficiency is achieved by a proper combination of load-stepping control technique and iterative solver for the nonlinear equilibrium equations. A major issue is the computation of the consistent tangent matrix, which is non-trivial due to nonlocal interaction between Gauss points. With computational efficiency in mind, we also present a new nonlocal damage model based on the nonlocal average of displacements. For this new model, the consistent tangent matrix is considerably simpler to compute than for current models. The various ideas discussed in the paper are illustrated by means of three application examples: the uniaxial tension test, the three-point bending test and the single-edge notched beam test.
\end{abstract}

Key words: quasi-brittle materials; nonlocal damage models; adaptivity; error estimation; consistent tangent matrix; quadratic convergence; nonlocal displacements

ऋ Research supported by Ministerio de Ciencia y Tecnología under grant DPI20012204

* Correspondence to: Antonio Rodríguez-Ferran, Departament de Matemàtica Aplicada III, E.T.S. de Ingenieros de Caminos, Canales y Puertos, Universitat Politècnica de Catalunya, Jordi Girona 1, E-08034 Barcelona, SPAIN.

Email address: antonio.rodriguez-ferran@upc.es (A. Rodríguez-Ferran).

$U R L$ : www-lacan.upc.es (A. Rodríguez-Ferran). 


\section{Introduction}

Damage models are nowadays a common choice in the numerical modelling of failure of quasi-brittle materials [1]. To avoid the pathological mesh dependence exhibited by local damage models, one may use either gradient damage models or nonlocal damage models. These two related strategies regularize the problem and ensure mesh objectivity. In gradient damage models, strain derivatives are incorporated into the constitutive equation [2]. In nonlocal damage models $[3,4,5]$, strain (or, rather, a strain-related state variable) is smoothed by means of an integral average in the vicinity - associated to a characteristic length - of each point. The latter approach is considered in this paper.

Another clear trend in computational mechanics is the quest for reliable computations. The quality of the results must be guaranteed in a quantifiable, objective manner. This has led to adaptive finite element analysis based on error estimation.

The price to pay for reliable results is a large number of degrees of freedom, especially in nonlinear solid mechanics. This means that one needs computationally efficient numerical methods.
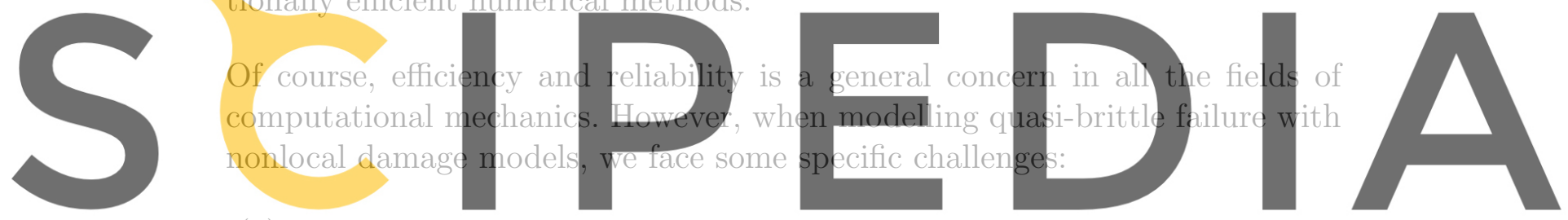

(1) Due to brittleness, the structural response is strongly nonlinear, very

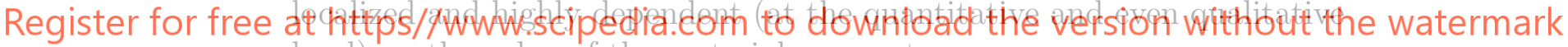
level) on the value of the material parameters.

(2) Due to nonlocality, there is interaction between non-adjacent finite elements. This poses several difficulties. The consistent tangent stiffness matrix, for instance (needed for quadratic convergence in Newton iterations), cannot be assembled from elementary contributions solely.

(3) Many error estimators are based on local (element-wise) computations. This fact must be conciliated with the nonlocal nature of the damage model: adaptivity typically leads to element sizes smaller than characteristic length.

\subsection{Objectives}

In this context, the main goal of this paper is to present an efficient and reliable approach for the numerical modelling of failure with nonlocal damage models. The key ingredients are: 
(1) A residual-type error estimator based on element-wise computations which, at the same time, accounts for the nonlocality of the constitutive model [6].

(2) An $h$-adaptive strategy driven by the error estimator which yields numerical results with the desired accuracy. The FE discretization errors are kept under control and, thus, the physical significance of the computations is guaranteed $[7,6]$.

(3) Advanced arc-length control techniques, adapted to the highly localized failure patterns.

(4) A flexible approach to achieve quadratic convergence in Newton iterations. The element-to-element stiffness matrices can either be assembled into the global tangent stiffness matrix [8] or accounted for in the righthand-side vector to prevent fill-in.

(5) A new model based on nonlocal displacements. The standard approach is to define the nonlocal state variable as the nonlocal average (NLA) of the (strain-related) local state variable. Other approaches have been proposed in the literature (see [9] for a comparative analysis), based, for instance, on nonlocal strains or nonlocal damage. An alternative approach is presented here: to use nonlocal displacements, obtained as the NLA of local displacements, to drive the evolution of damage. According to our preliminary numerical experiments, the resulting model exhibits a satis-

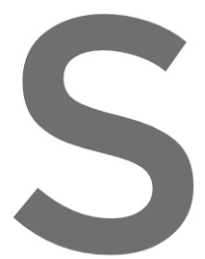

factory beh of view, especially matrix.
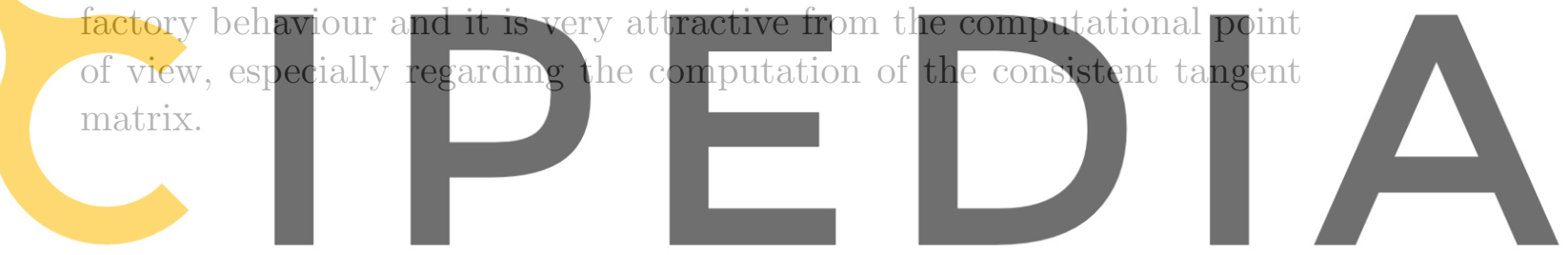

Register for free at https//www.scipedia.com to download the version without the watermark

1.2 Outline of Paper

The rest of the paper is organized as follows. Nonlocal damage models are briefly reviewed in Sect. 2. The proposed model based on nonlocal displacements is presented in Sect. 3 and illustrated with a uniaxial tension test. After that, various relevant issues regarding the efficiency and reliability of the computations are discussed in Sect. 4 and illustrated by means of numerical examples. Sect. 4.1 deals with iterative solvers, a key aspect for computational efficiency (numerical example: three-point bending test). The consistent tangent matrix for the proposed model based on nonlocal displacements is derived in Sect. 4.2 (uniaxial tension test). The adaptive strategy based on error estimation is discussed in Sect. 4.3 (single-edge notched beam test). The two main aspects, nonlinearity and nonlocality, are covered respectively in Sects. 4.3.1 and 4.3.2. The concluding remarks of Sect. 5 close the paper. 


\section{Overview of Nonlocal Damage Models}

For simplicity, only elastic-scalar damage models are considered here. However, many of the ideas, methods and algorithms can be extended to more complex damage models incorporating, for instance, anisotropy or plasticity $[5,10]$.

A generic nonlocal model of such type consists of the following equations, summarized in Table 1:

- A relation between Cauchy stresses $\sigma$ and small strains $\varepsilon$, where the loss of stiffness (from elastic stiffness $\mathbf{C}$ to zero stiffness) is described by means of a scalar damage parameter $D$ which ranges from 0 to $1,(1)$;

- The definition of a local state variable $Y$ as a function of strain $\varepsilon,(2)$;

- The definition of the nonlocal state variable $\tilde{Y}$ as the average of the local state variable $Y,(3)$ :

- A weighting function $\alpha$ which depends on the distance $r$ between two points and contains a characteristic length $l_{\mathrm{c}}$ as a parameter, (4);

- A damage evolution law, where the nonlocal state variable $\tilde{Y}$ drives the evolution of the non-decreasing damage parameter $D$, (5).

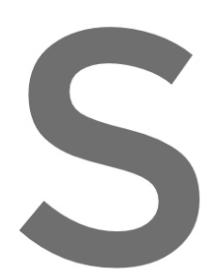

Table 1
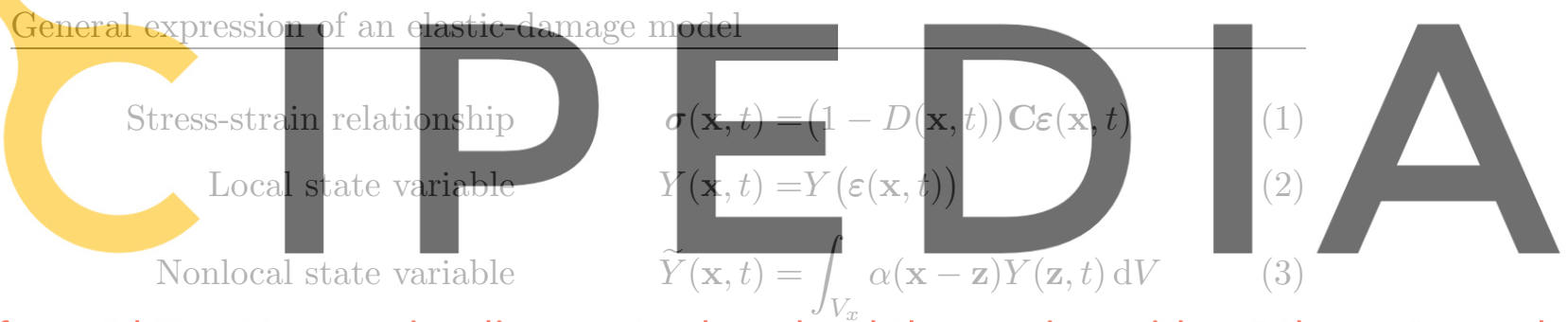

Register for free at https//www.scipedia.com

to download the version without the

watermark

$$
\text { Damage evolution } \quad D(\mathbf{x}, t)=D\left(\max _{\tau \leq t} \tilde{Y}(\mathbf{x}, \tau)\right)
$$

Many nonlocal damage models encountered in the literature can be accommodated with little or no modification into the general framework of Table 1. The most common choices for (2), (4) and (5) are reviewed next.

\subsection{Local State Variable}

The local state variable $Y$ is a suitable scalar measure of strains $\varepsilon$. Three common definitions are the energy release rate $[1,11]$

$$
Y=\frac{1}{2} \varepsilon^{T} \mathbf{C} \varepsilon,
$$


the average of positive principal strains $\varepsilon_{i}$ used in the Mazars model [12]

$$
Y=\sqrt{\sum_{i}\left[\max \left(0, \varepsilon_{i}\right)\right]^{2}}
$$

and a function of strain invariants used in the modified von Mises model [13]

$$
Y=\frac{k-1}{2 k(1-2 \nu)} I_{1}+\frac{1}{2 k} \sqrt{\left(\frac{k-1}{1-2 \nu} I_{1}\right)^{2}+\frac{12 k}{(1+\nu)^{2}} J_{2}} .
$$

In (8), $I_{1}$ and $J_{2}$ are the first and second invariants of the strain and deviatoric strain tensors respectively, and $k$ is the ratio of compressive to tensile strength.

\subsection{Weighting Function}

\section{The weighting function $\alpha$ is typically defined as}

$$
\alpha\left(r ; l_{c}\right)=\frac{\alpha_{0}\left(r ; l_{c}\right)}{\int_{V_{x}} \alpha_{0}\left(r ; l_{c}\right) d V}
$$
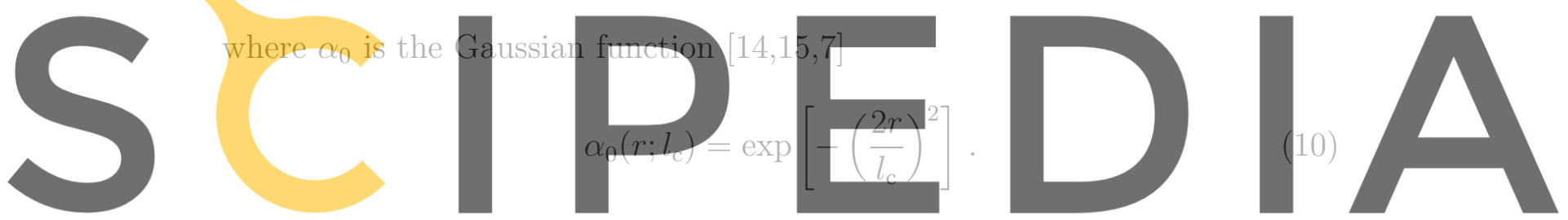

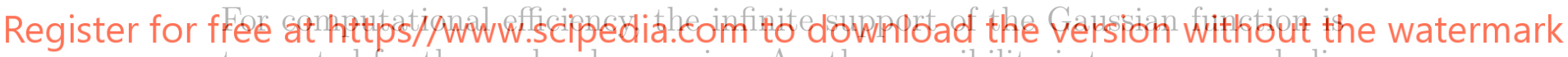
truncated for the nonlocal averaging. Another possibility is to use a parabolic function with compact support, see [9]. In any case, the integral in the denominator of (9) is not a constant: near the boundaries, the support of $\alpha_{0}$ may lay partially outside the domain, so a lower value of the integral is obtained. In fact, it is necessary to modify the Gaussian function $\alpha_{0}$ into the weighting function $\alpha$ as indicated by (9) to ensure consistency of order 0 (i.e. reproducibility of constant functions). This guarantees that a constant field of local state variable $Y(\mathbf{x})=Y$ is not modified due to nonlocal averaging (that is, $\tilde{Y}(\mathbf{x})=Y(\mathbf{x})=Y$ ) and, hence, that a constant strain field $\varepsilon$ results in a constant stress field $\boldsymbol{\sigma}$.

As a remark, is is worth noting that this function is sometimes written as $[9,16]$

$$
\alpha_{0}\left(r ; l_{\mathrm{c}}\right)=\exp \left[-\left(\frac{r}{\sqrt{2} l_{\mathrm{c}}}\right)^{2}\right] .
$$

Note that the characteristic lengths in (10) and (11) differ by a factor of $2 \sqrt{2}$. 


\subsection{Damage Evolution Law}

Two typical choices to describe the evolution of damage above the damage threshold $Y_{0}$ are the exponential law [12]

$$
D=1-\frac{Y_{0}(1-A)}{\tilde{Y}}-A \exp \left[-B\left(\tilde{Y}-Y_{0}\right)\right]
$$

and the polynomial law $[17,16]$

$$
D=1-\frac{1}{1+B\left(\tilde{Y}-Y_{0}\right)+A\left(\widetilde{Y}-Y_{0}\right)^{2}}
$$

In (12) and (13), parameter $A$ is associated to residual strength and parameter $B$ controls the slope of the softening branch at the peak (i.e. at $\widetilde{Y}=Y_{0}$ ), see $[7]$.

In Mazars model, damage $D$ is expressed as a combination of tensile damage $D_{t}$ and compressive damage $D_{c}[12]$. Each of these two components evolves according to an exponential law (12), with the corresponding parameters $A_{c}$

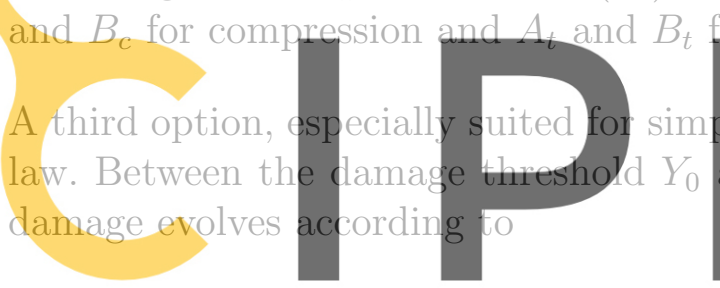

$D=\frac{Y_{\mathrm{f}}}{\gamma}$
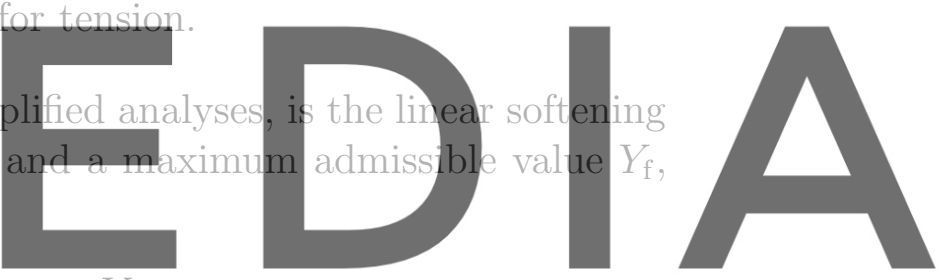

Register for free at https//www.scipedia.¿đm to dowmload the version without the watermark

which leads to a linear softening branch in a stress-strain diagram.

\section{A Nonlocal Damage Model Based on Nonlocal Displacements}

As Table 1 reflects, the standard approach is to define a scalar local state variable $Y$ (as a function of strains) and then to average it into the nonlocal state variable $\tilde{Y}$, which drives the evolution of damage.

However, other variables can be selected for averaging. In fact, a number a proposals can be found in the literature. Either scalar (for instance: damage $D$ ) or vectorial (for instance: strain $\varepsilon$ ) Gauss-point quantities may be averaged into the corresponding nonlocal quantities $(\widetilde{D}$ and $\widetilde{\varepsilon}$ in the two examples mentioned). The existing approaches are compared in [9] by means of a simple 1D numerical test (bar under uniaxial tension). 
Table 2

Standard approach (nonlocal state variable) vs. alternative approach (nonlocal displacements). Subscript NL denotes quantities with nonlocal information but computed locally. The tilde is reserved to truly nonlocal quantities (i.e. computed via nonlocal average, NLA, of a local quantity)

\begin{tabular}{ll}
\hline Standard approach & Alternative approach \\
\hline Local displacement $\mathbf{u}$ & Local displacement $\mathbf{u}$ \\
Local strain $\varepsilon=\nabla^{s} \mathbf{u}$ & Nonlocal displacement $\widetilde{\mathbf{u}}=\mathrm{NLA}(\mathbf{u})$ \\
Local state var. $Y=Y(\varepsilon)$ & Nonlocal strain $\varepsilon_{\mathrm{NL}}=\nabla^{s} \widetilde{\mathbf{u}}$ \\
Nonlocal state var. $\widetilde{Y}=\mathrm{NLA}(Y)$ & Nonlocal state var. $Y_{\mathrm{NL}}=Y\left(\varepsilon_{\mathrm{NL}}\right)$ \\
Damage evolution $D=D(\widetilde{Y})$ & Damage evolution $D=D\left(Y_{\mathrm{NL}}\right)$ \\
& Local strain $\varepsilon=\nabla^{s} \mathrm{u}$ \\
Stress-strain law $\sigma=(1-D) \mathrm{C}: \varepsilon$ & Stress-strain law $\sigma=(1-D) \mathrm{C}: \varepsilon$
\end{tabular}

A new proposal is made here: to compute nonlocal displacements $\widetilde{\mathbf{u}}$ by averaging the local (i.e. standard) displacements u. These nonlocal displacements
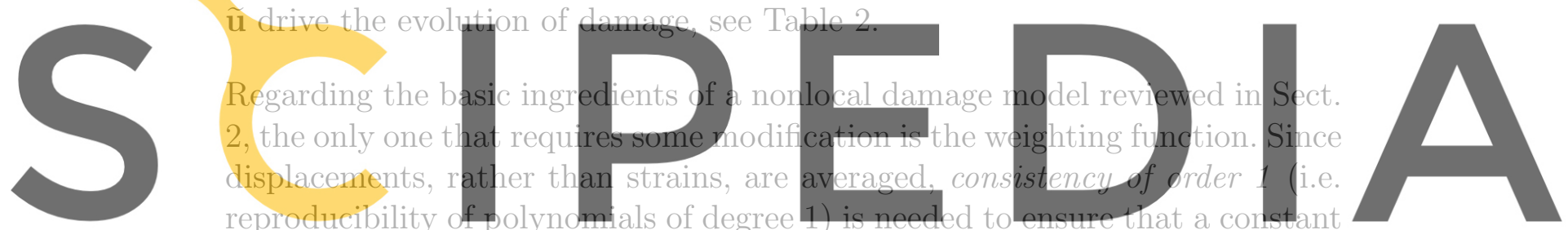

strain field results in a constant stress field. This can be done in a simple and

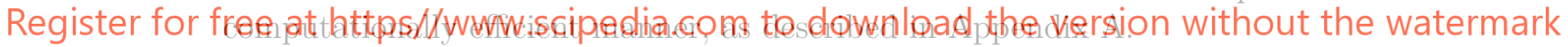

The relevance of consistency of order 1 is illustrated by Fig. 1. If a linear 1D field of local displacements is averaged with the weighting function (9), see Fig. 1(a), it is not reproduced correctly near the boundaries. The discrepancy increases with nonlocality, measured as the ratio of characteristic length to element size, see zoom in Fig. 1(b). With consistency of order 1, on the other hand, the nonlocal displacement field matches the local field, see Fig. 1(c).

Numerical Example: Uniaxial Tension Test

A clamped bar is subjected to uniaxial tension, see Fig. 2(a) and [18]. The onedimensional version of the damage model based on nonlocal displacements, see Table 2, with $Y(\varepsilon)=\varepsilon$ and a linear softening law, see Fig. 2(b), is used. The 


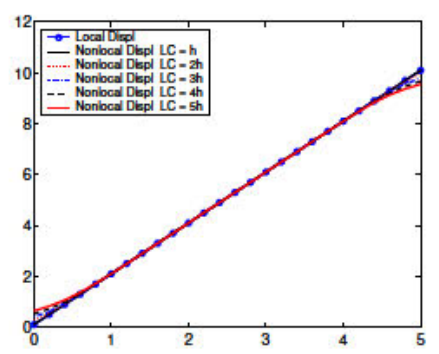

(a)

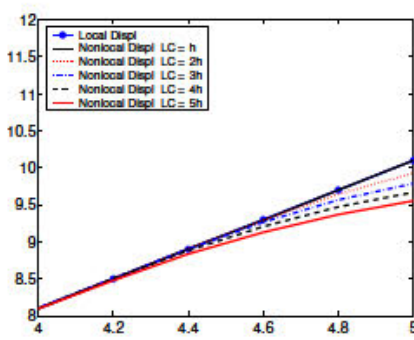

(b)

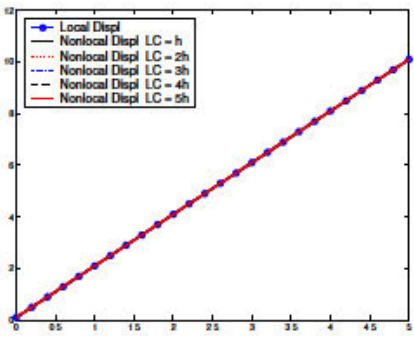

(c)

Figure 1. Nonlocal average of a linear function. Consistency of order 0: (a) whole domain; (b) zoom in boundary. (c) Consistency of order 1

central tenth of the bar is weakened ( $10 \%$ reduction in Young's modulus) to trigger localization. The dimensionless geometric and material parameters are summarized in Table 3. The numerical tests are displacement-controlled.
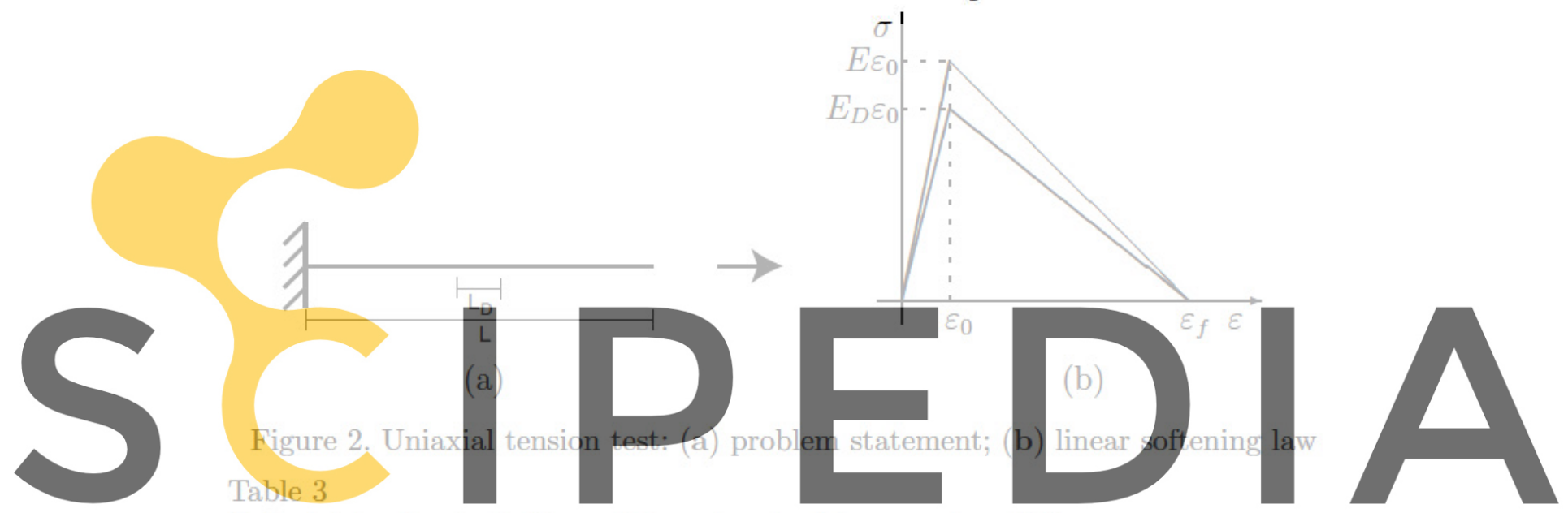

Uniaxial tension test. Geometric and material parameters [18]

Register for free at https//www:sicipedia.com tos downloadithe version without the watermark

\begin{tabular}{lll}
\hline Length of bar & $L$ & 100 \\
Idem of weaker part & $L_{D}$ & 10 \\
Young's modulus & $E$ & 20000 \\
Idem of weaker part & $E_{D}$ & 18000 \\
Damage threshold & $\varepsilon_{0}$ & $10^{-4}$ \\
Final strain & $\varepsilon_{f}$ & $1.25 \times 10^{-2}$ \\
\hline
\end{tabular}

The regularization properties of the model based on nonlocal displacements are assessed by carrying out the analysis with five different meshes of 40,80 , 160,320 and 640 elements (corresponding respectively to element sizes $h$ of $2.5,1.25,0.625,0.3125$ and 0.15625 ) and a fixed characteristic length $l_{\mathrm{c}}=6.25$ (corresponding respectively to $2.5,5,10,20$ and 40 elements). Both the forcedisplacement curves and the final damage profiles are virtually superimposed, see Fig. 3: mesh dependence is indeed precluded. 


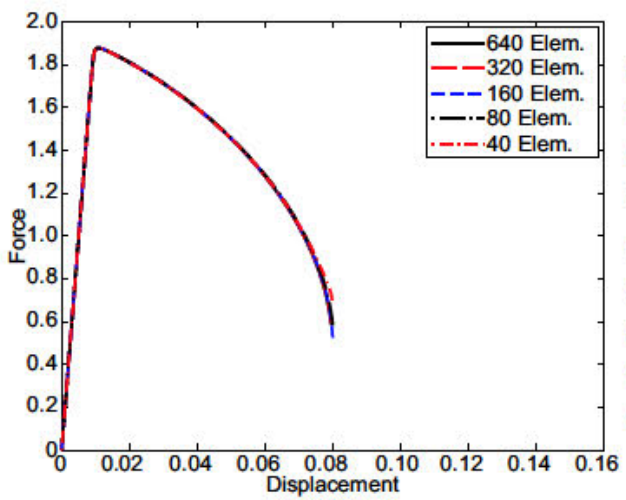

(a)

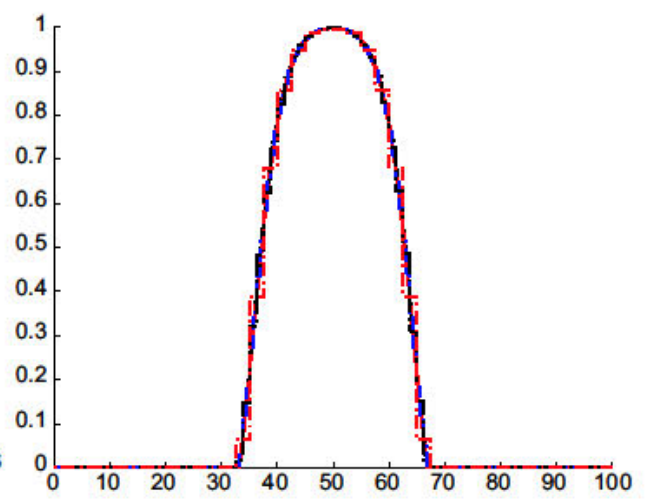

(b)

Figure 3. Uniaxial tension test. New model based on nonlocal displacements: (a) force-displacement and (b) final damage profiles for various meshes

\section{If, on the other hand, a fixed mesh of 320 elements $(h=0.3125)$ and four characteristic lengths of 5, 10, 20 and 40 elements are used, both the ductility in the force-displacement response and the width of the final damage profile increase, as expected, with the characteristic length, see Fig. 4.}
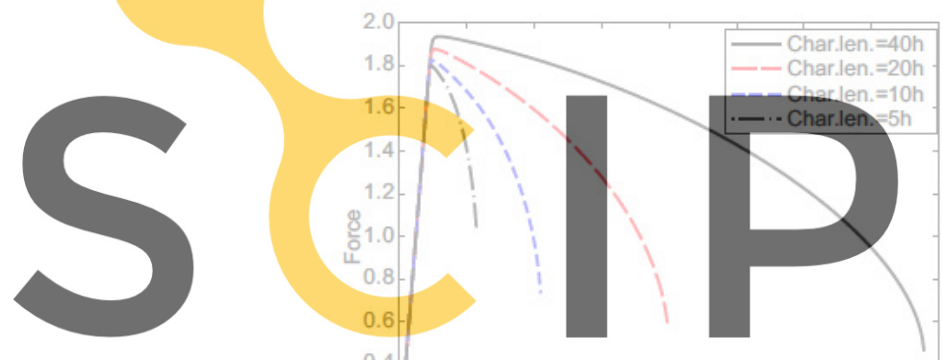

(a)

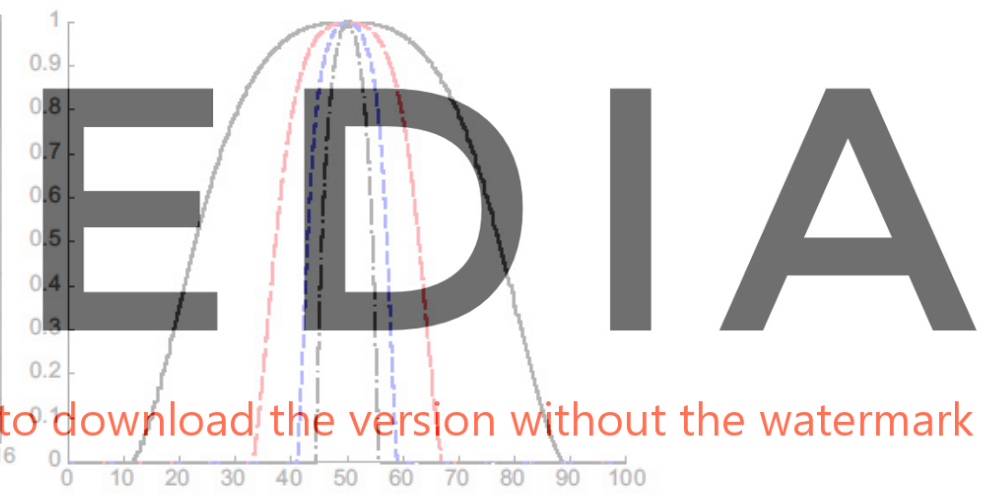

(b)

Figure 4. Uniaxial tension test. New model based on nonlocal displacements: (a) force-displacement and (b) final damage profiles for various characteristic lengths

To sum up: the new model exhibits the same regularization capabilities and qualitative response than the standard model based on nonlocal strains, depicted in Figs. 5 and 6.

A close look at Figs. 3 and 4 (for nonlocal displacements) and Figs. 5 and 6 (their counterparts for nonlocal strains) reveals that the quantitative agreement is also excellent. This point is further illustrated in Fig. 7, where the case of 320 elements and $l_{\mathrm{c}}=6.25$ is analyzed in detail. Apart from the same structural response, see Fig. 7(a), the two models also predict the same evolution of damage profile, see Fig. 7(b), and the same stress-strain diagram for three sample points, see Fig. 7(c). 


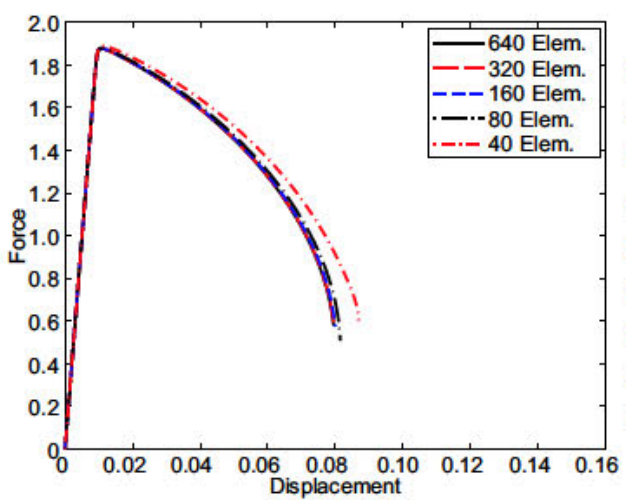

(a)

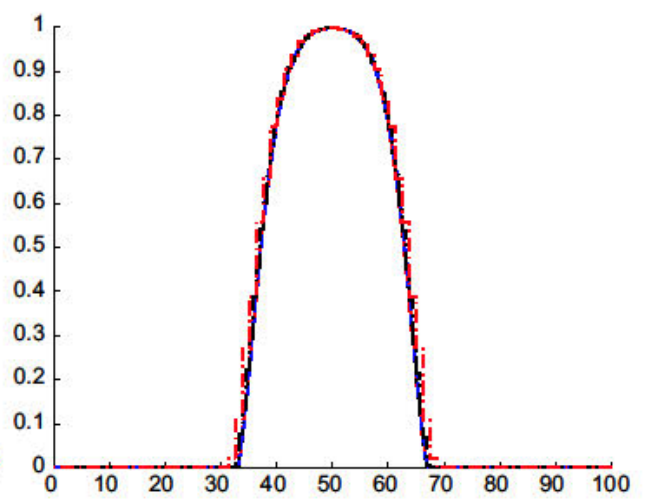

(b)

Figure 5. Uniaxial tension test. Standard model based on nonlocal strains: (a) force-displacement and (b) final damage profiles for various meshes
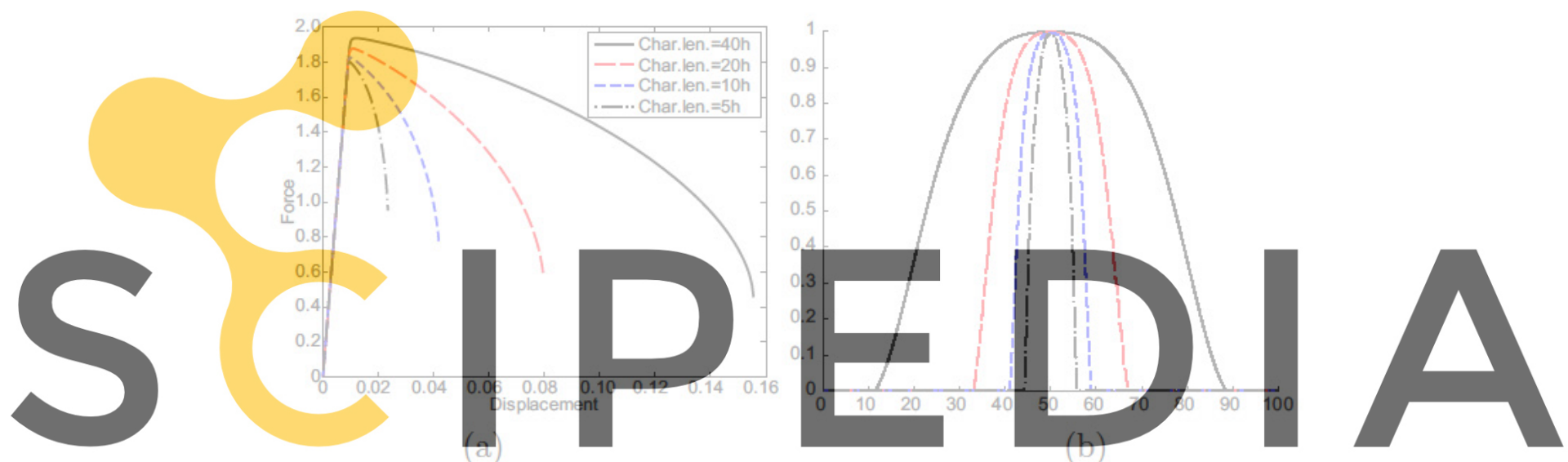

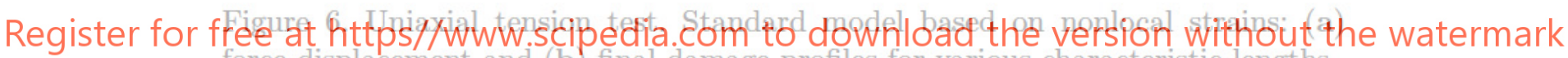
force-displacement and (b) final damage profiles for various characteristic lengths

\section{Efficiency and Reliability Issues}

As discussed in the introduction, reliability and efficiency are the two major issues in virtually any branch of computational (solid and fluid) mechanics. When modelling quasi-brittle materials, the development of reliable and efficient algorithms must take into account that the structural response is typically (1) very nonlinear, (2) localized, (3) highly dependent on the value of material parameters and (4) geometrically complex (curved cracks, primary and secondary failure mechanisms, etc.).

Various relevant issues regarding the efficiency and the reliability of the numerical simulations are discussed in separate Subsections and illustrated by means of a numerical example. 


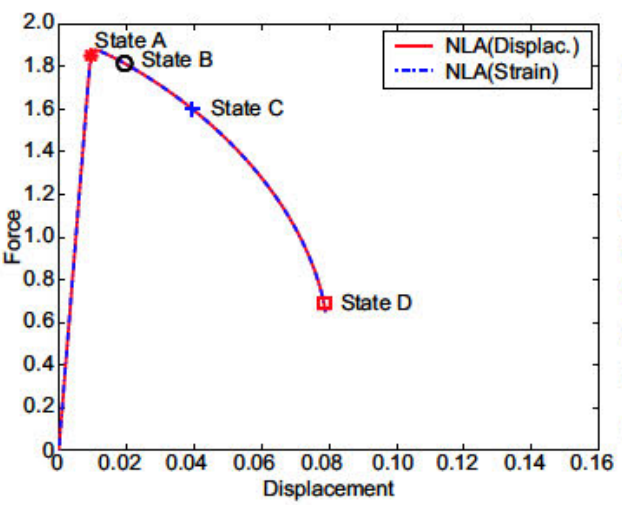

(a)
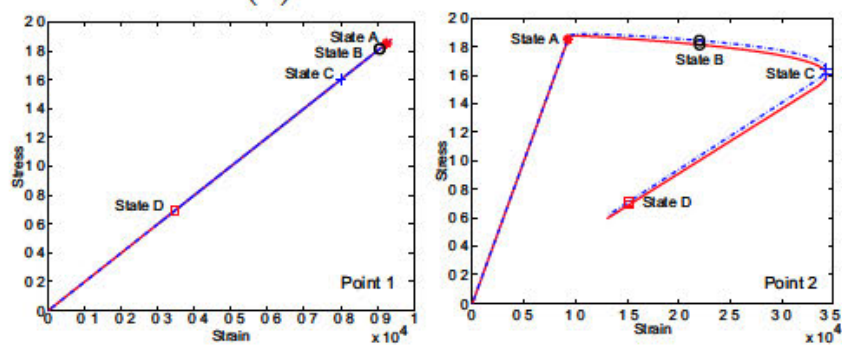

(c)

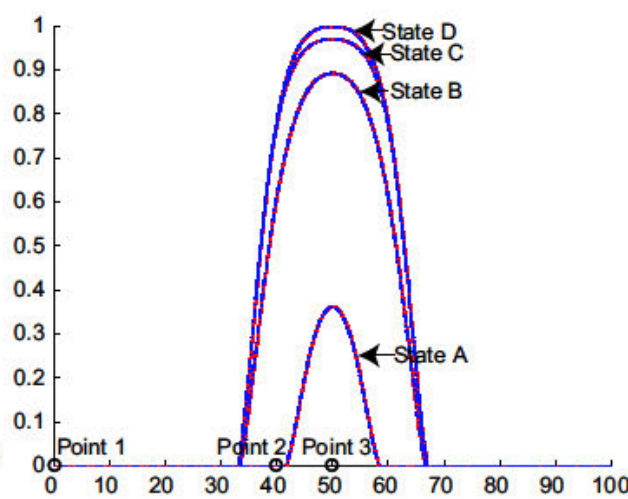

(b)

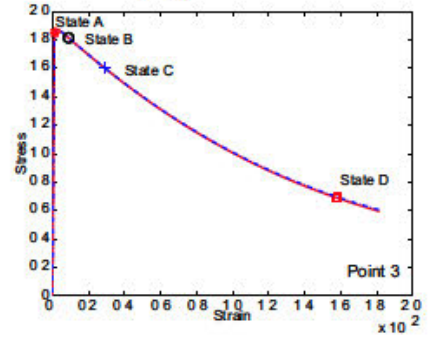

Figure 7. Uniaxial tension test. The new model (nonlocal displacements) and the standard model (nonlocal strains) yield very similar results: (a) force-displacement curve; (b) damage profiles; (c) stress-strain diagrams at sample points.

\subsection{Nonlinear Solvers and Quadratic Convergence}

Due to material nonlinearity, FE discretization of the equilibrium equation leads to a nonlinear system of algebraic equations, which is solved in an incremental-iterative fashion $[19,20]$. Thus, the two key ingredients for an efficient numerical model are:

(1) An adequate control strategy to define the increments (i.e. time- or loadstepping).

(2) A suitable nonlinear solver for the iterations within each increment, including the appropriate (secant or tangent) stiffness matrices.

Regarding the increments, force control is typically not a suitable control strategy, because the softening behaviour of the stress-strain law leads to structural softening of the snap-through or snap-back type. Instead, either displacement control (for snap-through) or arc-length control (for snap-through or snap-back) are required. In the latter case, the control parameter must be defined with care to account for the localized nature of quasi-brittle failure. For notched specimens, for instance, one can use the CMOD (Crack-Mouth Opening Displacement) or CMSD (Crack-Mouth Sliding Displacement) as the arc-length parameter. 
Within each increment, the equilibrium equation remains nonlinear and demands an iterative solution. A nonlinear solver amounts basically to the selection of a particular stiffness matrix for iterations.

\subsubsection{Iteration Matrices}

One possibility $[17,15,7,6]$ is to work with the secant stiffness matrix, computed from the damaged elastic moduli $(1-D) \mathbf{C}$. The main advantage of this approach is that the secant matrix is symmetric positive definite and very simple to compute (the factor $(1-D)$ at each Gauss point is the only difference with respect to the elastic stiffness matrix). The main drawback is that it must be supplemented with convergence acceleration and, even so, convergence is only linear.

If quadratic convergence is desired (full Newton-Raphson method), the consistent tangent matrix is required [20]. For nonlocal damage models, this poses a substantial difficulty: due to nonlocality, there is interaction between nonadjacent nodes, and the consistent tangent matrix exhibits a larger bandwidth (with respect to the sparsity pattern of the elastic or secant matrices) $[17,8]$, as discussed next.

In $\mathrm{FE}$ analysis, the internal force vector is typically computed with a Gauss quadrature as

$$
\mathbf{f}^{\text {int }}(\mathbf{u})=\sum_{p} w_{p} \mathbf{B}_{p}^{T} \boldsymbol{\sigma}_{p}(\mathbf{u})
$$

where $p$ ranges the Gauss points, $w_{p}$ are the corresponding integration weights, $\mathbf{B}_{p}$ is the usual matrix of shape function derivatives at Gauss point $p$ and stresses $\boldsymbol{\sigma}_{p}$ are

$$
\boldsymbol{\sigma}_{p}(\mathbf{u})=\left(1-D_{p}\right) \mathbf{C} \underbrace{\mathbf{B}_{p} \mathbf{u}}_{\varepsilon_{p}} .
$$

The consistent tangent matrix is

$$
\mathbf{K}_{\mathrm{tan}}:=\frac{\partial \mathbf{f}^{\mathrm{int}}}{\partial \mathbf{u}}=\sum_{p} w_{p} \mathbf{B}_{p}^{T} \frac{\partial \sigma_{p}}{\partial \mathbf{u}}
$$

Combining (16) and (17) results in

$$
\mathbf{K}_{\text {tan }}=\mathbf{K}_{\text {sec }}+\mathbf{K}_{\text {nonlocal }}
$$

where

$$
\mathbf{K}_{\mathrm{sec}}=\sum_{p} w_{p} \mathbf{B}_{p}^{T}\left(1-D_{p}\right) \mathbf{C B}_{p}
$$


is the secant stiffness matrix and

$$
\mathbf{K}_{\text {nonlocal }}=-\sum_{p} w_{p} \mathbf{B}_{p}^{T} \mathbf{C} \varepsilon_{p} \frac{\partial D_{p}}{\partial \mathbf{u}}
$$

is the nonlocal tangent contribution which accounts for the variation of the damage parameter.

By applying the chain rule, the term $\partial D_{p} / \partial \mathbf{u}$ can be expressed as

$$
\frac{\partial D_{p}}{\partial \mathbf{u}}=D^{\prime}\left(\tilde{Y}_{p}\right) \frac{\partial \tilde{Y}_{p}}{\partial \mathbf{u}}
$$

The integral (3) required for nonlocal averaging is also approximated via a numerical quadrature, so the nonlocal state variable $\widetilde{Y}_{p}$ is

$$
\tilde{Y}_{p}=\sum_{q \in V_{p}} w_{q} \alpha_{p q} Y_{q}
$$

where $q$ ranges the Gauss points $\boldsymbol{\xi}_{q}$ in the neighbourhood $V_{p}$ of Gauss point $\xi_{p}$, and $\alpha_{p q}=\alpha\left(r=\left\|\xi_{p}-\xi_{q}\right\|\right)$.

By differentiating (22), the last term in (21) can be expressed as

$$
\frac{\partial \tilde{Y}_{p}}{\partial \mathbf{u}}=\sum_{q \in V_{p}} w_{q} \alpha_{p q} \frac{\partial Y_{q}}{\partial \mathbf{u}}=\sum_{q \in V_{p}} w_{q} \alpha_{p q} \frac{\partial Y_{q}}{\partial \varepsilon} \mathbf{B}_{q}
$$

where the chain rule and the relation $\partial \varepsilon_{q} / \partial \mathbf{u}=\mathbf{B}_{q}$ have been used.

By replacing (23) into (21) and then into (20), the nonlocal matrix can be expressed as

$$
\mathbf{K}_{\text {nonlocal }, Y}=-\sum_{p, q \in V_{p}} w_{p q} \mathbf{B}_{p}^{T} \mathbf{C} \varepsilon_{p} D^{\prime}\left(\tilde{Y}_{p}\right) \frac{\partial Y_{q}}{\partial \varepsilon} \mathbf{B}_{q}
$$

where $w_{p q}=w_{p} w_{q} \alpha_{p q}$ and the subscript $Y$ denotes the nonlocal quantity. Due to the double loop in Gauss points caused by nonlocal interaction, $\mathbf{K}_{\text {nonlocal, } Y}$ cannot be assembled from elementary contributions solely.

To avoid the additional non-zero entries, some authors $[13,16]$ neglect the nonlocal interaction by taking $w_{p q}=0$ for $p \neq q$ :

$$
\mathbf{K}_{\text {local }, Y}=-\sum_{p} w_{p p} \mathbf{B}_{p}^{T} \mathbf{C} \varepsilon_{p} D^{\prime}\left(\tilde{Y}_{p}\right) \frac{\partial Y_{p}}{\partial \varepsilon} \mathbf{B}_{p}
$$

However, the resulting local tangent matrix $\mathbf{K}_{\mathrm{sec}}+\mathbf{K}_{\mathrm{local}, Y}$ is no longer consistent, and quadratic convergence is lost. 
Table 4

Properties of stiffness matrices

\begin{tabular}{|c|c|c|c|c|c|}
\hline & Matrix & Symmetry & $\begin{array}{l}\text { Increased } \\
\text { bandwidth }\end{array}$ & $\begin{array}{l}\text { Nonlocal } \\
\text { interaction }\end{array}$ & Convergence \\
\hline $\mathbf{K}_{\mathrm{sec}}$ & Secant & Yes & No & No & Linear \\
\hline $\mathbf{K}_{\mathrm{sec}}+\mathbf{K}_{\text {local }, \mathrm{I}}$ & Y Local tangent & No & No & No & Linear \\
\hline $\mathbf{K}_{\text {tan }}$ & Consistent tangent & No & Yes & Yes & Quadratic \\
\hline
\end{tabular}

These three basic choices are summarized in Table 4 .

\subsubsection{Quadratic Convergence Without Fill-in}

If the consistent tangent matrix is chosen, equilibrium iterations read

$$
\mathbf{K}_{\tan }^{i} \delta \mathbf{u}^{i+1}=-\mathbf{r}^{i}
$$

where $i$ is the iteration counter, $\mathbf{r}^{i}$ is the residual and $\delta \mathbf{u}^{i+1}$ is the iterative correction in displacements.

Due to the increased bandwidth of $\mathbf{K}_{\mathrm{tan}}^{i}$, fill-in during the factorization is considerably larger than for a local (tangent or secant) stiffness matrix. If this additional fill-in is a critical factor, it can be avoided by accounting for the nonlocal interaction in the right-hand-side vector. The consistent tangent matrix can be expressed as

$$
\mathbf{K}_{\mathrm{tan}}=\mathbf{K}_{\mathrm{sec}}+\mathbf{K}_{\mathrm{local}, Y}+\mathbf{K}_{p \neq q, Y}
$$

where $\mathbf{K}_{p \neq q, Y}$ is the part of the nonlocal matrix $\mathbf{K}_{\text {nonlocal }, Y}$ neglected when approximating (24) by (25).

Equations (26) and (27) can be combined into the system of equations

$$
\left(\mathbf{K}_{\mathrm{sec}}^{i}+\mathbf{K}_{\mathrm{local}, Y}^{i}\right) \delta \mathbf{u}^{i+1}=-\mathbf{r}^{i}-\mathbf{K}_{p \neq q, Y}^{i} \delta \mathbf{u}^{i+1}
$$

which can be solved with an inner iterative scheme,

$$
\left(\mathbf{K}_{\mathrm{sec}}^{i}+\mathbf{K}_{\mathrm{local}, Y}^{i}\right) \delta \mathbf{u}_{k+1}^{i+1}=-\mathbf{r}^{i}-\mathbf{K}_{p \neq q, Y}^{i} \delta \mathbf{u}_{k}^{i+1}
$$

where $k$ is the counter for the inner iterations. Note that extra fill-in is indeed precluded, because the matrix in system (29) is local. Moreover, once $\mathbf{K}_{\mathrm{sec}}^{i}+\mathbf{K}_{\text {local }, Y}^{i}$ is factorized, the inner iterations have a relatively modest computational cost. Linear convergence is expected for these inner iterations $k$, but - and this is the key issue -, quadratic convergence without increased fill-in will be achieved for the expensive, outer equilibrium equations $i$. Moreover, the tolerance of the inner $k$ loop is usually not taken as a constant, but 


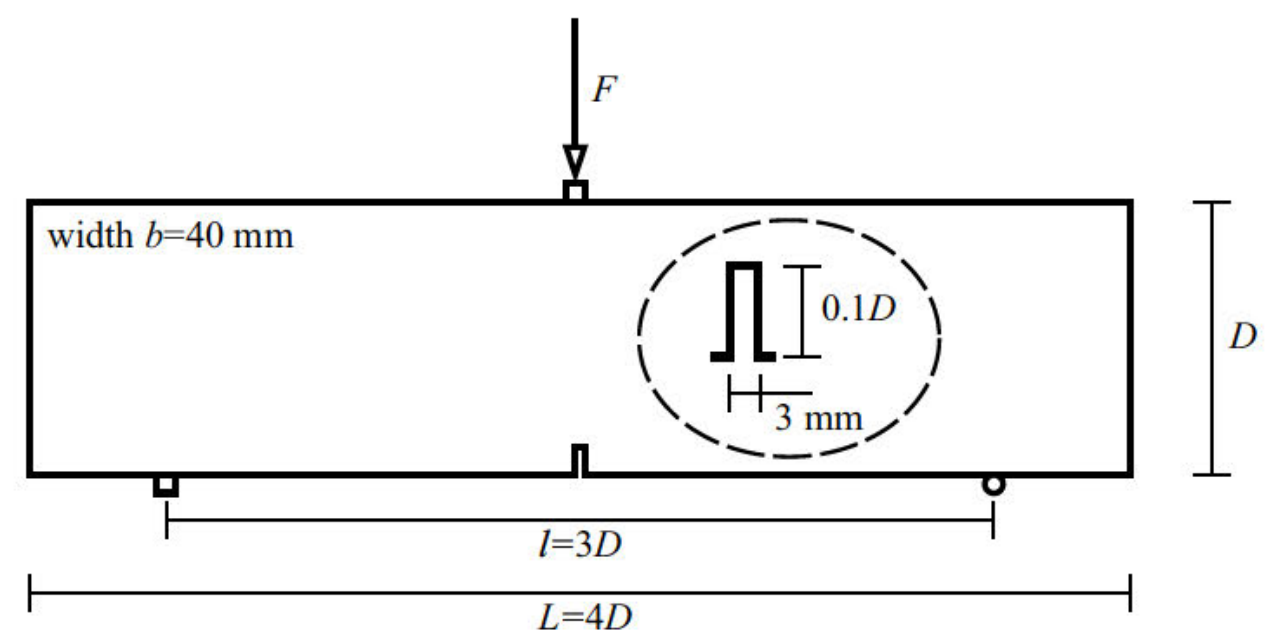

Figure 8. Three-point bending test. Problem statement (Size $\mathrm{D}=320 \mathrm{~mm}$ )

dependent on outer iteration $i$ to increase the efficiency. This is standard in numerical algebra [21]. In fact at the initial iterations of the Newton-Raphson method a larger tolerance is prescribed and, as $i$ increases, the tolerance of the inner loop approaches the standard value.

If factorizing the non-symmetric matrix $\mathbf{K}_{\mathrm{sec}}^{i}+\mathbf{K}_{\text {local }, Y}^{i}$ is inconvenient with the finite element code at hand, matrix $\mathbf{K}_{\text {local, } Y}^{i}$ can be taken to the righthand-side. The inner loop then reads

$$
\mathbf{K}_{\mathrm{sec}}^{i} \delta \mathbf{u}_{k+1}^{i+1}=-\mathbf{r}^{i}-K_{\text {nonlocal }, Y}^{i} \delta \mathbf{u}_{k}^{i+1},
$$

and the usual Cholesky factorization applies. More (but cheaper) inner iterations should be expected.

\section{Numerical Example: Three-Point Bending Test}

A notched beam is subjected to three-point bending, see Fig. 8. This test is modelled with the Mazars model [12] and the material parameters obtained in [22], see Table 5, by fitting the results of various experiments. Plane strain conditions are assumed.

The problem is solved with arc-length control, using the Crack Mouth Opening Displacement (CMOD) as the control variable. The structural response of Fig. 9 is obtained. Note that the force-deflection curve exhibits a certain amount of snap-back behaviour.

The convergence behaviour of the various nonlinear solvers is summarized in Fig. 10. Note that quadratic convergence is achieved with the consistent tangent matrix, while only linear convergence is obtained with the secant matrix. 
Table 5

Three-point bending test. Material parameters for the Mazars model [22]

\begin{tabular}{lll}
\hline Meaning & Symbol & Value \\
\hline Young's modulus & $E$ & $38500 \mathrm{MPa}$ \\
Poisson's coefficient & $\nu$ & 0.24 \\
Characteristic length & $l_{\mathrm{c}}$ & $40 \mathrm{~mm}$ \\
Damage threshold & $Y_{0}$ & $3 \times 10^{-5}$ \\
Parameter $A$ in compression & $A_{c}$ & 1.25 \\
Parameter $B$ in compression & $B_{c}$ & 1000 \\
Parameter $A$ in tension & $A_{t}$ & 0.95 \\
Parameter $B$ in tension & $B_{t}$ & 9000 \\
\hline
\end{tabular}

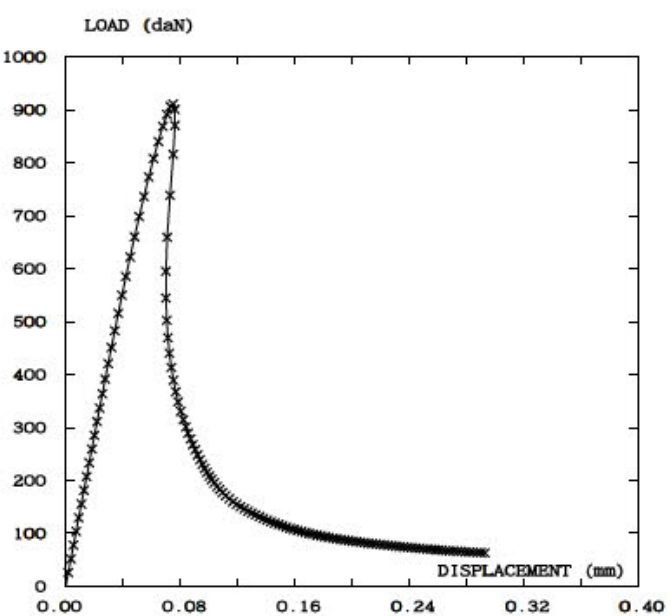

(a)

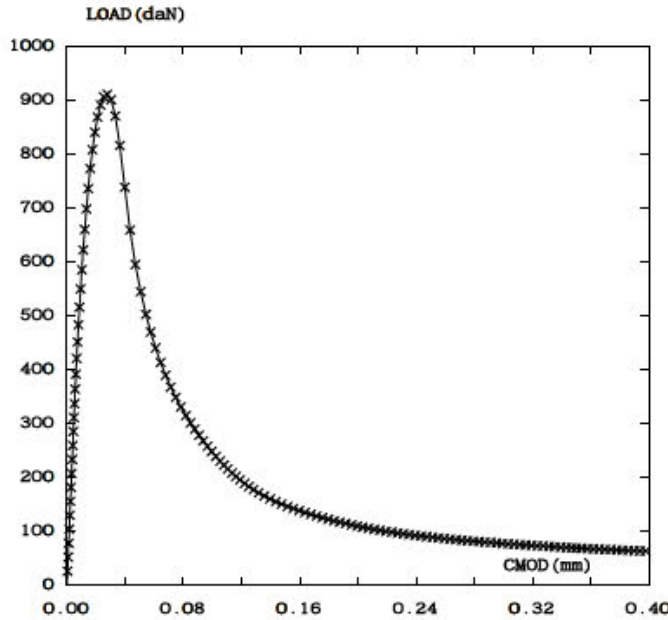

(b)

Figure 9. Three-point bending test. Structural response obtained with arc-length control: (a) force-vertical deflection; (b) force-CMOD (Crack Mouth Opening Displacement)
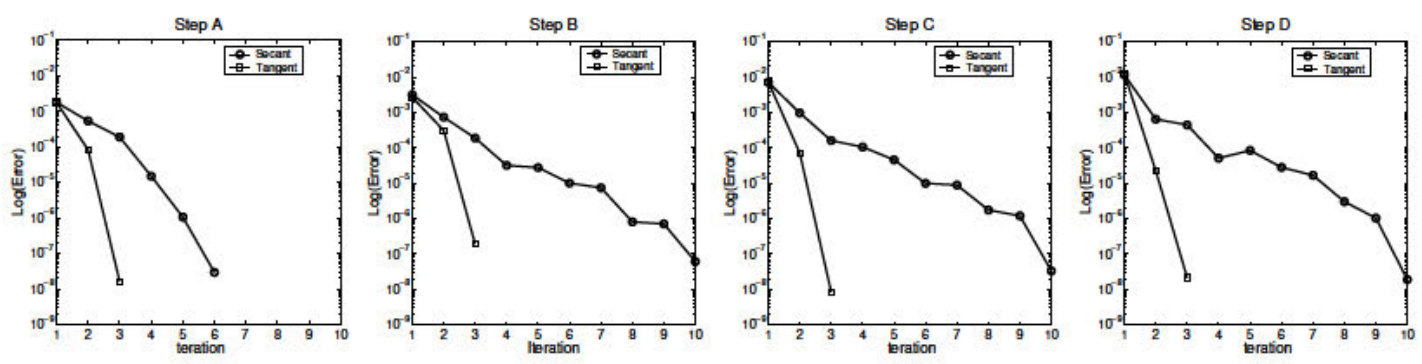

Figure 10. Three-point bending test. Convergence history for representative load-steps with the secant matrix and the consistent tangent matrix 


\subsection{Consistent Tangent Matrix for Model Based on Nonlocal Displacements}

The proposed model based on nonlocal displacements has very attractive numerical properties. As shown here, the consistent tangent matrix is quite simpler to compute than in the standard case shown above.

Equations (15)-(20) are also valid for the new model. However, the term $\partial D_{p} / \partial \mathbf{u}$ is now

$$
\frac{\partial D_{p}}{\partial \mathbf{u}}=D^{\prime}\left(Y_{\mathrm{NL}_{p}}\right) \frac{\partial Y}{\partial \varepsilon_{\mathrm{NL}}}\left(\varepsilon_{\mathrm{NL}_{p}}\right) \frac{\partial \varepsilon_{\mathrm{NL}_{p}}}{\partial \widetilde{\mathbf{u}}} \frac{\partial \widetilde{\mathbf{u}}}{\partial \mathbf{u}}
$$

Since nonlocal averaging is performed at the beginning, the rest of the constitutive model is "local". Note, in particular, that the usual shape functions are used in the FE discretization of nonlocal displacements and that nonlocal strains $\varepsilon_{\mathrm{NL}}$ are computed locally as the symmetrized gradient of nonlocal displacements, see Table 2. This means that

$$
\varepsilon_{\mathrm{NL}_{p}}=\mathbf{B}_{p} \widetilde{\mathbf{u}} \quad \Longrightarrow \quad \frac{\partial \varepsilon_{\mathrm{NL}_{p}}}{\partial \widetilde{\mathbf{u}}}=\mathbf{B}_{p}
$$

where $\mathbf{B}_{p}$ is the same matrix of shape function derivatives used in (15).

The last term in (31), $\partial \widetilde{\mathbf{u}} / \partial \mathbf{u}$, reflects the nonlocality of the model. After finite element discretization and numerical integration, the averaging process (A.6) leads simply to

$$
\widetilde{\mathbf{u}}=\mathbf{A} \mathbf{u} \quad \Longrightarrow \quad \frac{\partial \widetilde{\mathbf{u}}}{\partial \mathbf{u}}=\mathbf{A}
$$

where $\mathbf{A}$ is a matrix of nonlocal connectivity. Note that this matrix contains purely geometrical information associated to the finite element mesh. It does not change as damage evolves, so it can be computed and stored at the beginning of the analysis (provided, of course, that a fixed mesh is used).

Substitution of (31), (32) and (33) into (20) results in

$$
\mathbf{K}_{\text {nonlocal, }}=\mathbf{K}_{\text {local, } \mathbf{u}} \mathbf{A}
$$

with

$$
\mathbf{K}_{\text {local }, \mathbf{u}}=-\sum_{p} w_{p} \mathbf{B}_{p}^{T} \mathbf{C} \boldsymbol{\varepsilon}_{p} D^{\prime}\left(Y_{\mathrm{NL}_{p}}\right) \frac{\partial Y}{\partial \varepsilon_{\mathrm{NL}}}\left(\boldsymbol{\varepsilon}_{\mathrm{NL}_{p}}\right) \mathbf{B}_{p}
$$

Note that $\mathbf{K}_{\text {local,u }}$ can be computed in the usual way by assembling elementary matrices, like in any local material model. After that, nonlocality is accounted for by means of the constant matrix $\mathbf{A}$, which "spreads" the stiffness of $\mathbf{K}_{\text {local, }}$ into $\mathbf{K}_{\text {nonlocal, } \mathbf{u}}$. 
Step A

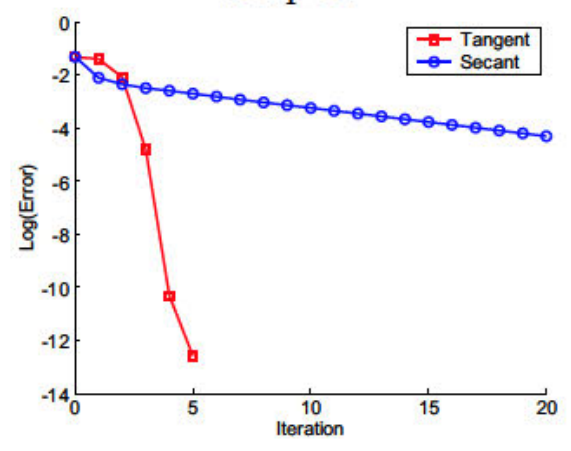

Step C

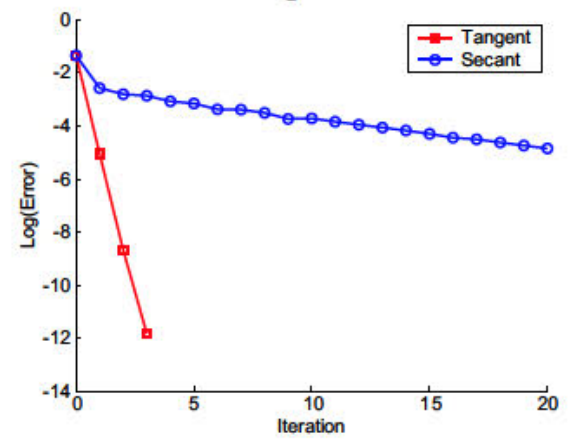

Step B

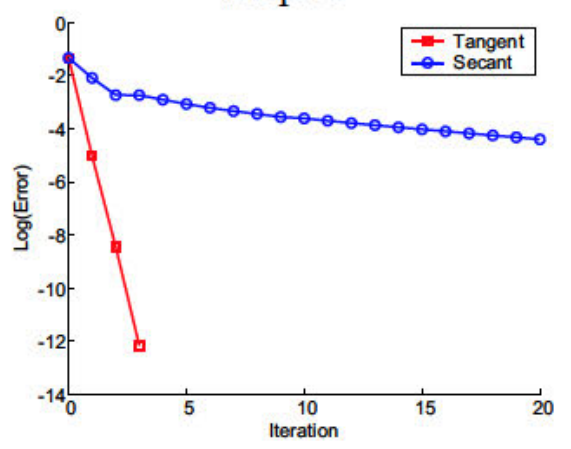

Step D

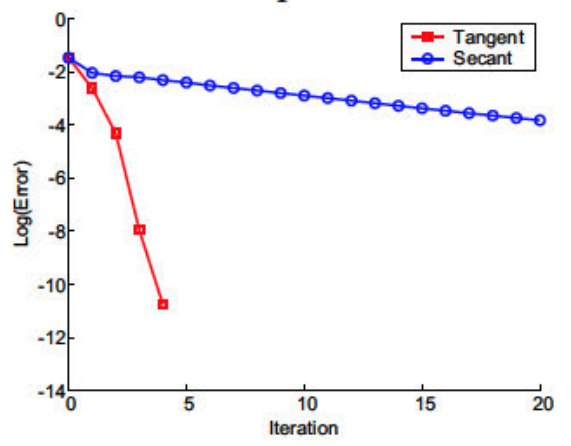

Figure 11. Uniaxial tension test. Convergence history of the secant matrix and the consistent tangent matrix for load steps A, B, C and D, see Fig. 7(a)

By replacing (34) in (18), the consistent tangent matrix can be expressed as

$$
\mathbf{K}_{\text {tan }}=\mathbf{K}_{\text {sec }}+\mathbf{K}_{\text {local }} \mathbf{A} \text {. }
$$

This simple structure of $\mathbf{K}_{\tan }$ is due to the fact that the nonlocal average is performed completely "upstream" in the constitutive equation (i.e. with displacements, the primal unknowns in the FE analysis).

\section{Numerical Example: Uniaxial Tension Test}

The uniaxial tension test of Sect. 3 is resumed here to illustrate the computation of the consistent tangent matrix for the model based on nonlocal displacements. The case with 320 elements and $l_{\mathrm{c}}=6.25$, see Fig. 7 , is considered. Figure 11 shows the convergence behaviour with two different nonlinear solvers at the four representative load steps depicted in Fig. 7(a). As expected, only linear convergence is achieved with the secant stiffness matrix, while the consistent tangent matrix leads to quadratic convergence.

The simple structure of the consistent tangent matrix (36) is graphically illustrated by Fig. 12. Note how the constant matrix A indeed "spreads" the local stiffness matrix $\mathbf{K}_{\text {local }}$ (which is non-zero only where damage increases), 

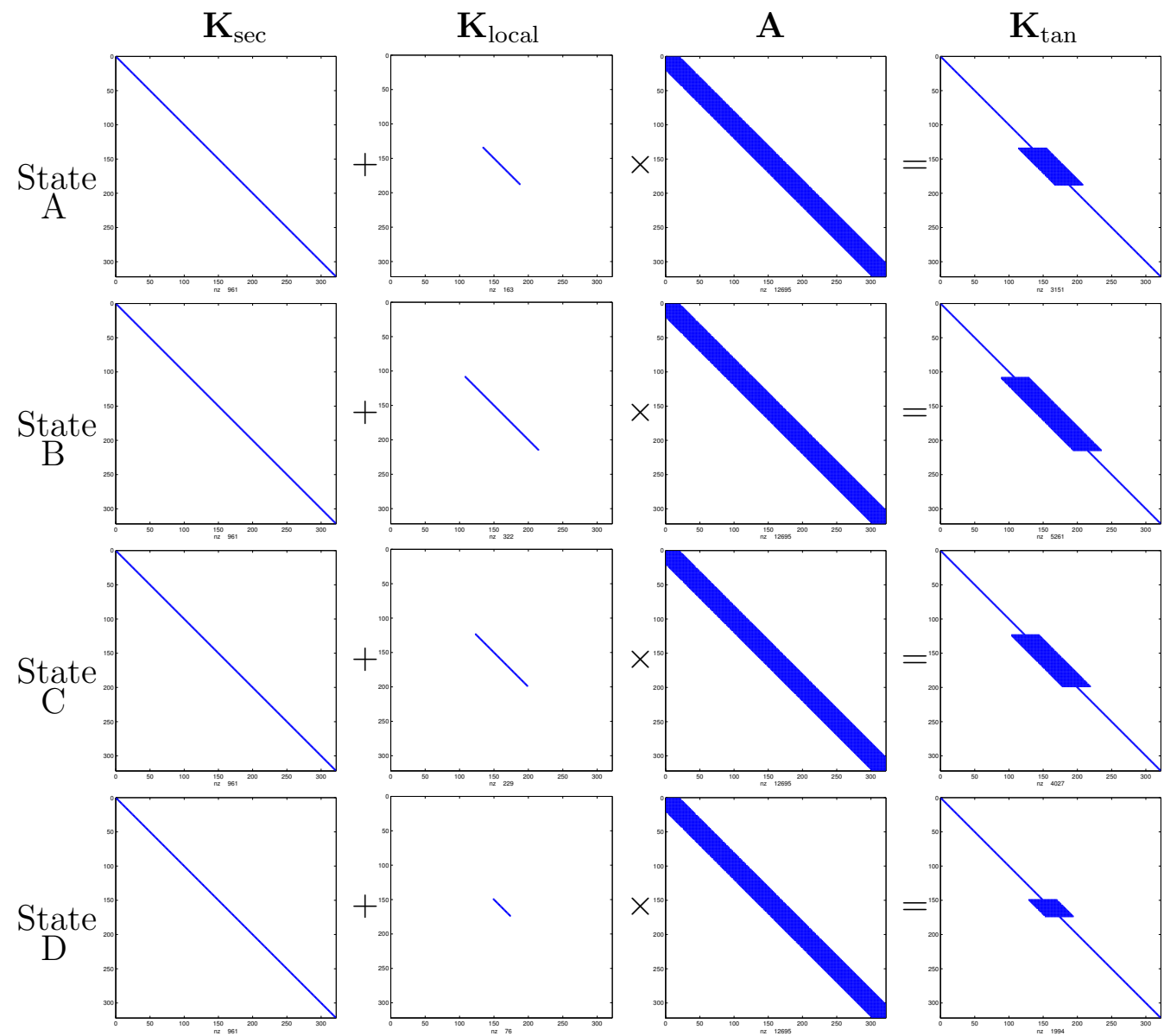

Figure 12. Uniaxial tension test. Sparsity pattern of the consistent tangent matrix at load steps A, B, C and D, see Fig. 7(a)

thus provoking fill-in in the tangent matrix $\mathbf{K}_{\tan }$.

\subsection{Adaptivity Based on Error Estimation}

Even for nonlinear problems, simply obtaining a finite element solution is nowadays not enough. One must also ensure the quality of the numerical results in an objective, quantifiable manner. With that purpose, we present here an adaptive strategy based on error estimation $[7,6]$. The two key ingredients are a residual-type error estimator for nonlinear problems [23] and $h$-remeshing $[24]$.

The issue of reliability is relevant in all fields of computational mechanics. In failure modelling of quasi-brittle materials, it is critical. Due to brittleness, the particular choice of a constitutive equation or a set of material parameters can have a very significant influence (not only quantitative but even qualitative) on the failure mechanism. 
Of course, the finite element mesh also affects the numerical solution. For this reason, it is essential to keep FE errors under control when assessing the effect of material modelling. If models or sets of parameters are compared with a given mesh (deemed "sufficiently fine" but with no objective measure of its quality), the effect of FE discretization errors could be erroneously attributed to the different material models.

The key ingredient of the adaptive strategy is the error estimator. We use a residual-type error estimator first developed for linear problems in continua [25] and later extended to other problems, such as local nonlinear models, e.g. plasticity or visco-plasticity [23,26], nonlocal nonlinear models (nonlocal damage [6]), or (linear and nonlinear) shells [27].

The focus here is in the two main issues of the problem under consideration: nonlinearity and nonlocality. More details about the error estimator can be found in the references just cited.

\subsubsection{Error Estimation: Nonlinearity}

Finite element discretization of the governing partial differential equation renders the algebraic nonlinear equilibrium equation

$$
\mathbf{f}_{H}^{\text {int }}\left(\mathbf{u}_{H}\right)=\mathbf{f}_{H}^{\text {ext }},
$$

where the unknown is the nodal displacement vector $\mathbf{u}_{H}, \mathbf{f}_{H}^{\text {int }}\left(\mathbf{u}_{H}\right)$ is the vector of nodal internal forces associated with $\mathbf{u}_{H}$ and $\mathbf{f}_{H}^{\text {ext }}$ is the discretized external force term. Subscript $H$ denotes that the working mesh has characteristic size $H$.

The exact error of $\mathbf{u}_{H}$ is defined as $\mathbf{e}_{\mathbf{u}}^{\text {exact }}:=\mathbf{u}-\mathbf{u}_{H}$, where $\mathbf{u}$ is the exact solution. Of course, $\mathbf{e}_{\mathbf{u}}^{\text {exact }}$ cannot be computed because $\mathbf{u}$ is not available. Instead, the error $\mathbf{e}_{\mathbf{u}}^{\text {exact }}$ is approximated by the reference error $\mathbf{e}_{\mathbf{u}}:=\mathbf{u}_{h}-\mathbf{u}_{H}$, where $\mathbf{u}_{h}$ is the finite element solution obtained with a finer mesh ( $h \ll H$, the approach considered here) or a higher-order interpolation:

$$
\mathbf{f}_{h}^{\text {int }}\left(\mathbf{u}_{h}\right)=\mathbf{f}_{h}^{\text {ext }} .
$$

Note that (38) can also be expressed as

$$
\mathbf{f}_{h}^{\text {int }}\left(\mathbf{u}_{H}+\mathbf{e}_{\mathbf{u}}\right)=\mathbf{f}_{h}^{\text {ext }} .
$$

Computing $\mathbf{e}_{\mathbf{u}}$ (or, equivalently, $\mathbf{u}_{h}$ ) is computationally much more expensive than computing $\mathbf{u}_{H}$, because it involves solving a much larger nonlinear system of equations over a finer mesh, (38) or (39). For this reason, the basic 

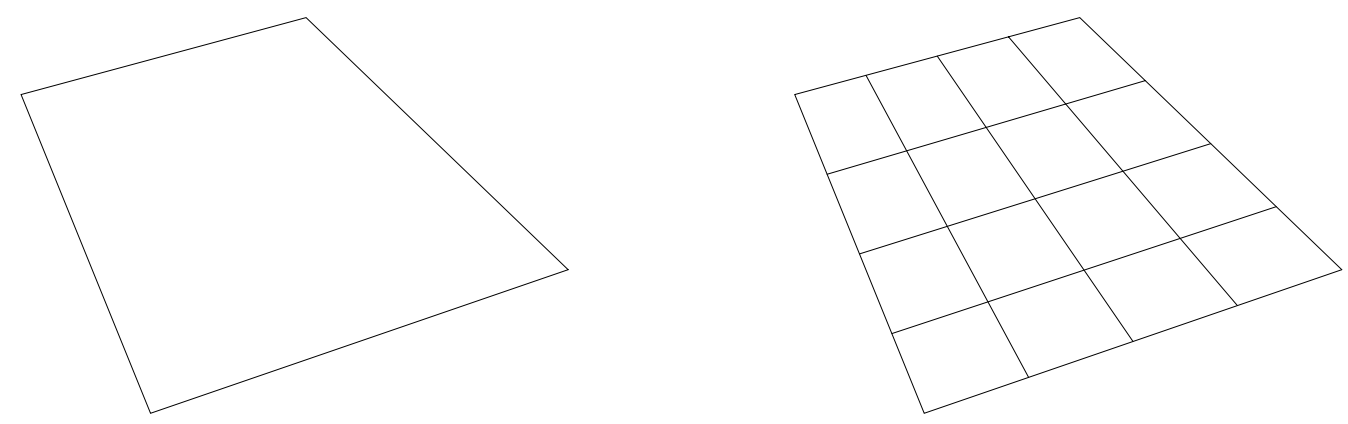

Figure 13. Finite element of mesh $H$ subdivided into $4 \times 4$ elements of mesh $h$

idea of residual-type error estimators is to approximate $\mathbf{e}_{\mathbf{u}}$ by low-cost local computations over subdomains.

The natural subdomains for local computations are the finite elements. For this reason, the first phase of the error estimator consists on solving the nonlinear system (39) locally inside each finite element of the working mesh (interior estimate, see Table 6). To do so, each element $\Omega_{k}$ of size $H$ is meshed into $4 \times 4$ elements of size $h$, see Fig. 13. That it, the fine mesh $h$ is nested into the working mesh $H$, with $h=H / 4$.

To avoid the expensive flux-splitting procedures of other residual-type error estimators (required to prescribe Neumann boundary conditions for each local problem), homogeneous Dirichlet boundary conditions for the error are prescribed on the element boundary $\partial \Omega_{k}$ (that is, $\mathbf{u}_{h}=\mathbf{u}_{H}$ on $\partial \Omega_{k}$ ). This equality is also set, over all the element $\Omega_{k}$, as the initial approximation. Once error $\mathbf{e}_{\text {elem }}^{k}$ is obtained, its squared energy norm (based on the SPD secant stiffness matrix) is computed and added up into the global error estimate.

Of course, the error $\mathbf{e}_{\mathbf{u}}$ is not really zero along all element edges, as assumed during the interior estimate. For this reason, a second set of local problems is solved, over a different partition of the computational domain into subdomains. A natural choice is to associate these subdomains, called patches, to the nodes of the working mesh (patch estimate, see Table 7). If four-noded quadrilateral elements are used, a patch consists of one-fourth of each element sharing the node, see Fig. 14.

To compute the estimate $\mathbf{e}_{\text {patch }}^{\ell}$ for patch $\Lambda_{\ell}$, the same ideas discussed for elements apply. Again, the boundary conditions and the initial approximation for the local nonlinear problem over the patch consist in setting $\mathbf{e}_{\text {patch }}^{\ell}$ to zero over $\partial \Lambda_{\ell}$ and $\Lambda_{\ell}$ respectively. The only difference is that orthogonality between patch estimate $\mathbf{e}_{\text {patch }}^{\ell}$ and interior estimate $\mathbf{e}_{\text {elem }}$ must be imposed, as discussed next.

The total error in patch $\Lambda_{\ell}$ is the sum of the patch estimate $\mathbf{e}_{\text {patch }}^{\ell}$ and the 
Table 6

Pseudocode for the first phase of the error estimation procedure. The interior estimate $\mathrm{E}$ is stored both locally $\left(\mathrm{E}\left(\Omega_{k}\right)\right.$ for $\left.k=1,2, \ldots\right)$ and globally $(\mathrm{E}(\Omega)$ for the whole domain $\Omega$ )

loop on elements $k=1,2 \ldots$

- Build up local refined mesh for element $\Omega_{k}$

- Set trivial Dirichlet b.c. $\mathbf{e}_{\text {elem }}^{k}=\mathbf{0}$ on $\partial \Omega_{k}$

- Set initial approximation $\mathbf{e}_{\text {elem }}^{k}=\mathbf{0}$ in $\Omega_{k}$

- Solve iteratively local nonlinear problem

$$
\mathbf{r}\left(\mathbf{e}_{\text {elem }}^{k}\right):=\left.\mathbf{f}_{h}^{\text {int }}\left(\mathbf{u}_{H}+\mathbf{e}_{\text {elem }}^{k}\right)\right|_{\Omega_{k}}-\left.\mathbf{f}_{h}^{\text {ext }}\right|_{\Omega_{k}}=\mathbf{0}
$$

- Compute squared local norm

$$
\mathrm{E}\left(\Omega_{k}\right)=\left(\mathbf{e}_{\text {elem }}^{k}\right)^{T} \mathbf{K}_{\mathrm{sec}, h}^{k} \mathbf{e}_{\text {elem }}^{k}
$$

- Store error function: $\mathbf{e}_{\text {elem }} \leftarrow \mathbf{e}_{\text {elem }}+\mathbf{e}_{\text {elem }}^{k}$

- Upgrade global estimate: $\mathrm{E}(\Omega) \leftarrow \mathrm{E}(\Omega)+\mathrm{E}\left(\Omega_{k}\right)$

end loop

Interior estimate

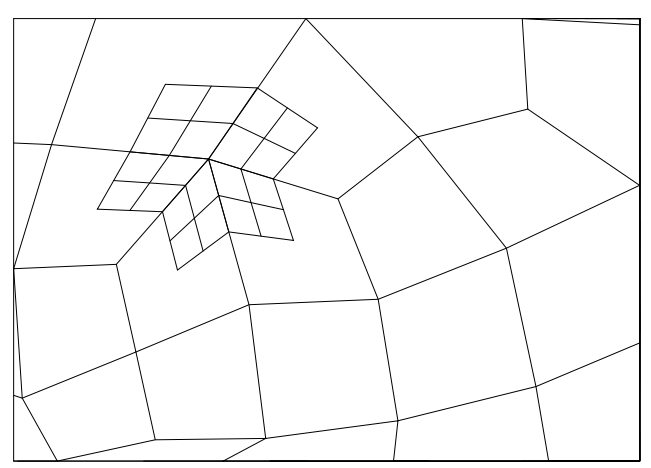

Figure 14. Patch associated to a node of mesh $H$ subdivided into $4 \times 4$ elements of mesh $h$

interior estimate restricted to the patch, $\mathbf{e}_{\text {elem }}^{\Lambda_{\ell}}$ :

$$
\mathbf{e}_{\text {total }}^{\Lambda_{\ell}}=\mathbf{e}_{\mathrm{patch}}^{\ell}+\mathbf{e}_{\mathrm{elem}}^{\Lambda_{\ell}} .
$$


The squared norm of the total error is

$$
\begin{aligned}
\mathrm{E}_{\text {total }}^{\Lambda_{\ell}} & =\left(\mathbf{e}_{\text {total }}^{\Lambda_{\ell}}\right)^{T} \mathbf{K}_{\mathrm{sec}, h}^{k} \mathbf{e}_{\text {total }}^{\Lambda_{\ell}} \\
& =\underbrace{\left(\mathbf{e}_{\mathrm{patch}}^{\ell}\right)^{T} \mathbf{K}_{\mathrm{sec}, h}^{k} \mathbf{e}_{\mathrm{patch}}^{\ell}}_{\mathrm{E}\left(\Lambda_{\ell}\right)}+\underbrace{\left(\mathbf{e}_{\mathrm{elem}}^{\Lambda_{\ell}}\right)^{T} \mathbf{K}_{\mathrm{sec}, h}^{k} \mathbf{e}_{\mathrm{elem}}^{\Lambda_{\ell}}}_{\mathrm{E}_{\text {elem }}^{\Lambda_{\ell}}}+\underbrace{2\left(\mathbf{e}_{\mathrm{elem}}^{\Lambda_{\ell}}\right)^{T} \mathbf{K}_{\mathrm{sec}, h}^{k} \mathbf{e}_{\mathrm{patch}}^{\ell}}_{0} .
\end{aligned}
$$

Note that orthogonality between $\mathbf{e}_{\text {elem }}^{\Lambda_{\ell}}$ and $\mathbf{e}_{\text {patch }}^{\ell}$ (i.e. cancellation of the third term in the RHS of (41)) is required so that the squared norm of the total error can be obtained by adding the squared norms of the interior and patch estimates.

The requirement $\left(\mathbf{e}_{\mathrm{elem}}^{\Lambda_{\ell}}\right)^{T} \mathbf{K}_{\mathrm{sec}, h}^{k} \mathbf{e}_{\mathrm{patch}}^{\ell}=0$ is a multi-point linear constraint on $\mathbf{e}_{\text {patch }}^{\ell}$. For convenience, it can be expressed as

$$
\mathbf{c}^{T} \mathbf{e}_{\text {patch }}^{\ell}=0 \quad \text { with } \quad \mathbf{c}=\mathbf{K}_{\text {sec }, h}^{k} \mathbf{e}_{\text {elem }}^{\Lambda_{\ell}}
$$

The linear constraint (42) can be imposed in a simple manner by means of Lagrange multipliers, see [28]. It is also possible to use so-called transformation methods, see references cited in [28].

The proposed two-phase approach for error estimation is summarized in Tables 67.

\subsubsection{Error Estimation: Nonlocality}

The proposed two-phase approach for error estimation consists basically in solving two sequences of local nonlinear problems over subdomains (elements and patches), see Tables 6 and 7. The material model, however, is nonlocal. As a consequence, the internal forces $\mathbf{f}^{\text {int }}$ must be carefully computed in order to account for the nonlocal nature of the damage model [6].

The "natural" approach would be, see central column of Table 8, to obtain the error in strains $\mathbf{e}_{\boldsymbol{\varepsilon}}$ from the error in displacements $\mathbf{e}_{\mathbf{u}}$ (in the corresponding element or patch), compute the refined strains $\varepsilon_{h}$ and the local state variable $Y_{h}$. The nonlocal average over the subdomain (element $k$ or patch $\ell$ ), NLA sub, then yields the nonlocal state variable $\widetilde{Y}_{h}$, which drives the damage parameter, $D_{h}$. Finally, refined stresses $\sigma_{h}$ are computed.

Note that the nonlocal average that transforms $Y_{h}$ into $\tilde{Y}_{h}$ is over a local support. This fact leads to non-physical responses, especially in zones of large damage gradients. Assume, for instance, that the error in strains is small and $\varepsilon_{h} \approx \varepsilon_{H}$. A small variation in $\tilde{Y}$ is also expected $\left(\widetilde{Y}_{h} \approx \widetilde{Y}_{H}\right)$. However, it 
Table 7

Pseudocode for the second phase of the error estimation procedure. The patch estimate is used to improve the estimate both locally $\left(\mathrm{E}\left(\Omega_{k}\right)\right.$ for $\left.k=1,2, \ldots\right)$ and globally $(\mathrm{E}(\Omega)$ for the whole domain $\Omega)$

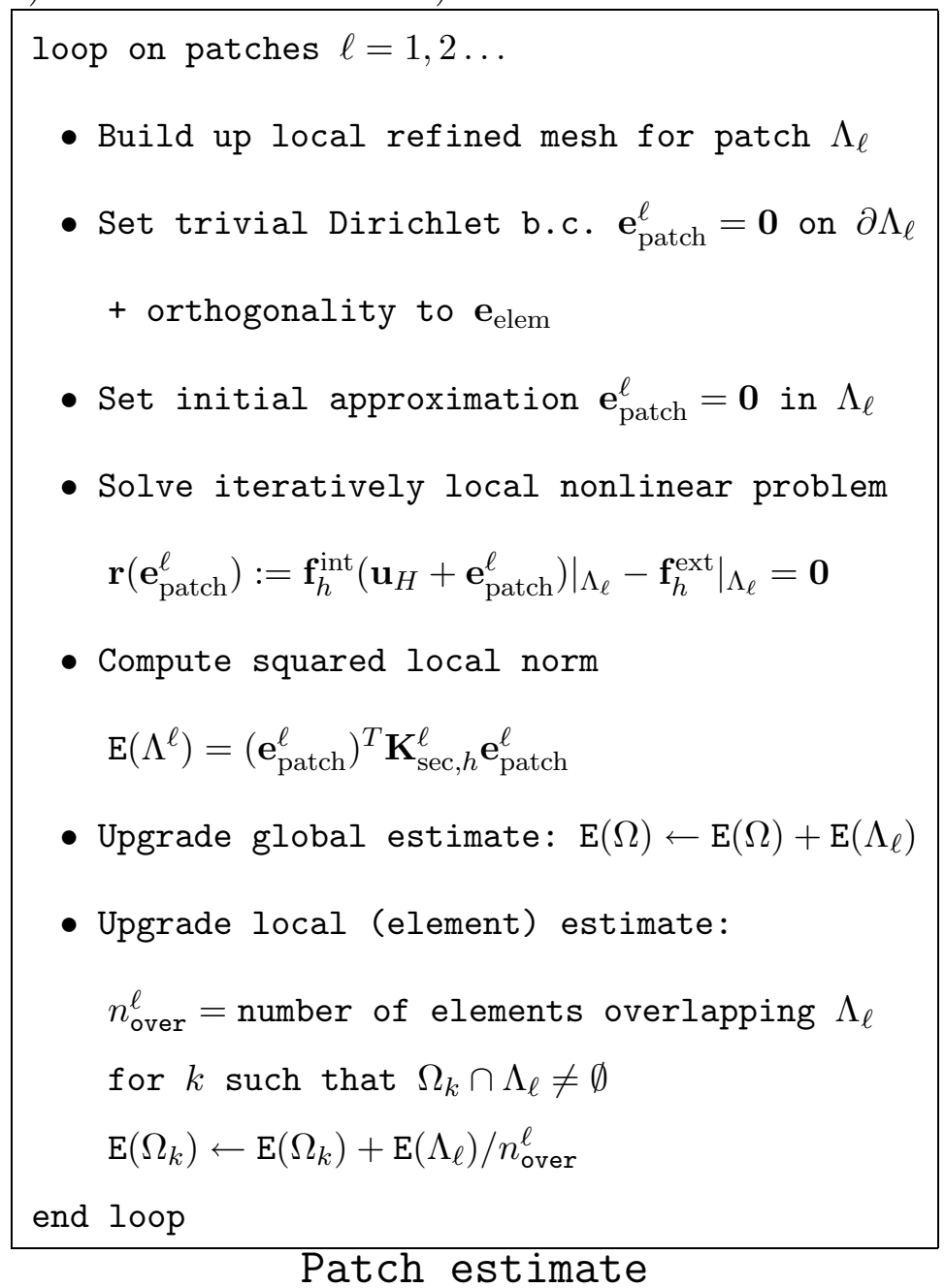

may happen that $\tilde{Y}_{h} \ll \tilde{Y}_{H}$, because $\tilde{Y}_{h}$ contains no information about nearby zones.

This point is illustrated in Fig. 15, which depicts the local state variable, the nonlocal state variable and the damage parameter for a given time increment in a zone of the working mesh $H$ with large gradients. The circled element has a very small local state variable $Y_{H}$, see Fig. 15(a), below the threshold $Y_{0}$. However, since the elements to the right have large values of $Y_{H}$, it has a relatively large (above $Y_{0}$ ) nonlocal state variable $\widetilde{Y}_{H}$, see Fig. $15(\mathrm{~b})$, which leads to damage, see Fig. 15(c). If the standard model is used to solve the local problem on the circled element during error estimation, a small error in strains leads to a small variation in the local state variable which, after nonlocal averaging over the element, results in a low value of the nonlocal state variable (that is, $\widetilde{Y}_{h} \ll \widetilde{Y}_{H}$ ). As a consequence, damage cannot increase 
Table 8

Standard nonlocal damage model and model modified for error estimation. Note the difference in the computation of the nonlocal state variable, steps 4 and 5

\section{Standard model Model for error estimation}

\begin{tabular}{lll}
\hline 1. Error in displ. & $\mathbf{e}_{\mathbf{u}}$ & $\mathbf{e}_{\mathbf{u}}$ \\
2. Error in strains & $\mathbf{e}_{\varepsilon}=\nabla^{s}\left(\mathbf{e}_{\mathbf{u}}\right)$ & $\mathbf{e}_{\varepsilon}=\nabla^{s}\left(\mathbf{e}_{\mathbf{u}}\right)$ \\
3. Strain & $\varepsilon_{h}=\varepsilon_{H}+\mathbf{e}_{\varepsilon}$ & $\varepsilon_{h}=\varepsilon_{H}+\mathbf{e}_{\varepsilon}$ \\
4. Local state var. & $Y_{h}=Y\left(\varepsilon_{h}\right)$ & $Y_{h} \approx Y_{H}+\underbrace{\frac{\partial Y}{\partial \varepsilon}\left(\varepsilon_{H}\right) \mathbf{e}_{\varepsilon}}$ \\
& & \\
5. Nonlocal state var. & $\widetilde{Y}_{h}=\operatorname{NLA}_{\text {sub }}\left(Y_{h}\right)$ & $e_{\widetilde{Y}}=\operatorname{NLA}_{\text {sub }}\left(e_{Y}\right) \quad ;$ \\
6. Damage & & $\widetilde{Y}_{h}=\widetilde{Y}_{H}+e_{\widetilde{Y}}$ \\
7. Stresses & $D_{h}=D\left(\widetilde{Y}_{h}\right)$ & $D_{h}=D\left(\widetilde{Y}_{h}\right)$ \\
& $\sigma_{h}=\left(1-D_{h}\right) \mathbf{C}: \varepsilon_{h}$ & $\sigma_{h}=\left(1-D_{h}\right) \mathbf{C}: \varepsilon_{h}$ \\
\hline
\end{tabular}

in the circled element during error estimation. When estimating the error for the circled element, the nonlocal state variable $\tilde{Y}_{H}$, rather than the local state variable $Y_{H}$, is representative of its mechanical properties.

For this reason, the nonlocal damage model is slightly modified for error estimation, see right column in Table 8 . The difference resides in the way the nonlocal state variable $\widetilde{Y}_{h}$ is computed. By means of a first-order Taylor expansion, the local state variable $Y_{h}$ is expressed as $Y_{H}$ plus an error term $e_{Y}$. Note that the derivatives $\partial Y / \partial \varepsilon$ needed for computing $e_{Y}$ are also required for the computation of the consistent tangent matrix, so they do not represent a computational overhead of the modified model.

The error term $e_{Y}$ is averaged over the element/patch into $e_{\widetilde{Y}}$. As a consequence, $\tilde{Y}_{h}$ is computed as the addition of a reference value $\tilde{Y}_{H}$, which describes the real damaged stiffness, and an error term $e_{\widetilde{Y}}$.

With this modified model, a small variation in strains does result in a small variation in the nonlocal state variable (that is, $\tilde{Y}_{h} \approx \widetilde{Y}_{H}$ ). Going back to Fig. 15, this means that the damage level of the circled element may either remain constant (for $\tilde{Y}_{h}<\tilde{Y}_{H}$ ) or increase (for $\tilde{Y}_{h}>\tilde{Y}_{H}$ ) during error estimation.

To sum up: the standard model is not capable of capturing the spread of the damaged zone associated to error estimation. 


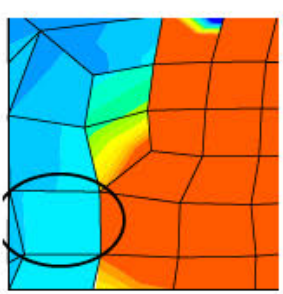

(a)

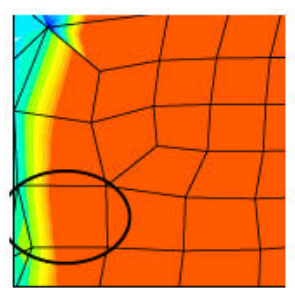

(b)

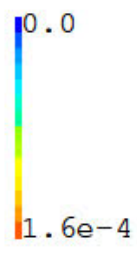

)

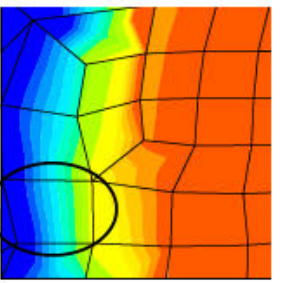

(c)

Figure 15. Fields in a zone of large gradients: (a) local state variable $Y$; (b) nonlocal state variable $\widetilde{Y} ;\left(\right.$ c) damage. The damage threshold is $Y_{0}=1.5 \times 10^{-4}$

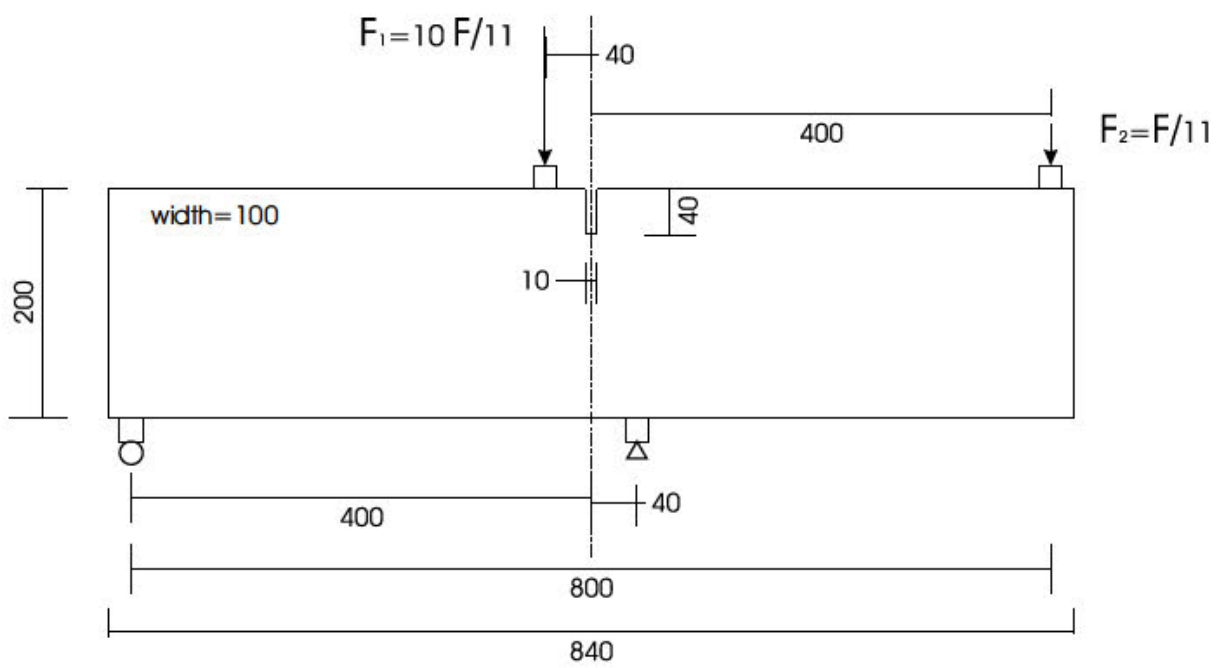

Figure 16. Single-edge notched beam. Problem statement (all distances in mm)

Numerical Example: Single-Edge Notched Beam Test

A single-edge notched beam (SENB) is subjected to anti-symmetrical fourpoint bending [29]. The geometry, loads and supports are shown in Fig. 16. A plane stress analysis is performed. The concrete beam is modelled with the modified von Mises nonlocal damage model [13] with exponential damage evolution, while the steel loading platens are assumed to be elastic. The material parameters for concrete are shown in Table 9. For steel, a Poisson's coefficient $\nu=0.2$ and a Young's modulus 10 times that of concrete are used.

The results of the adaptive process are shown in Figs. 17-19. The initial mesh is shown in Fig. 17(a). Note that this mesh is relatively coarse, with only one element in the notch width. The final damage distribution and deformed mesh (amplified 300 times), corresponding to a CMSD of $0.08 \mathrm{~mm}$, is depicted in Fig. 17(b). The curved crack pattern observed in experiments [29] is clearly captured. The error estimation procedure discussed in Sect. 4.3 is employed to compute the error field of Fig. 17(d). The error is larger in the damaged zone and near the loading platens. The global relative error (i.e. energy norm of the error in displacements over the energy norm of displacements) is $3.96 \%$, 
Table 9

SENB test. Material parameters for the modified von Mises model [6]

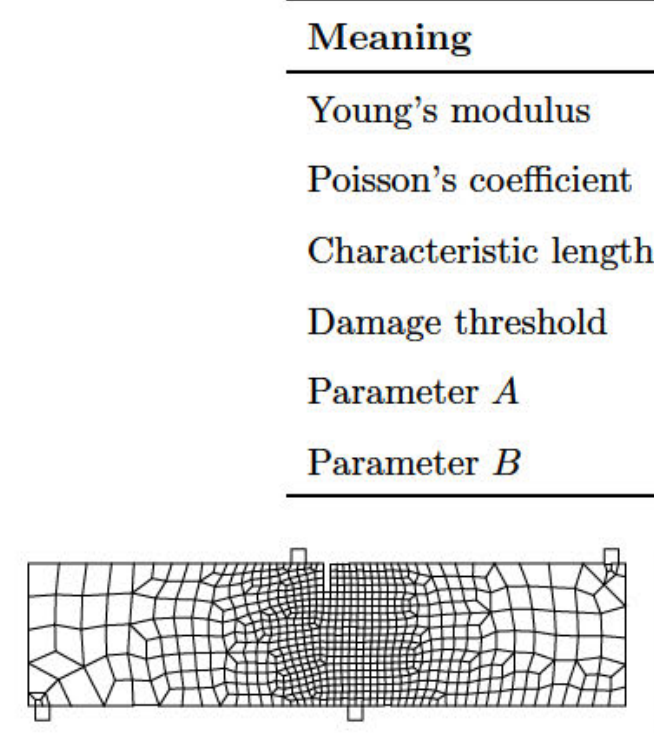

(a)

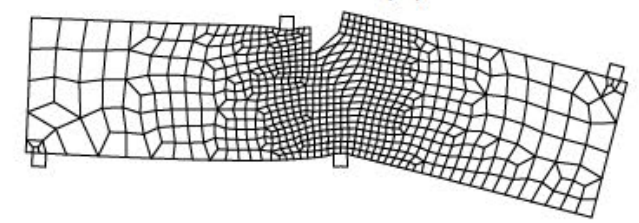

(c)

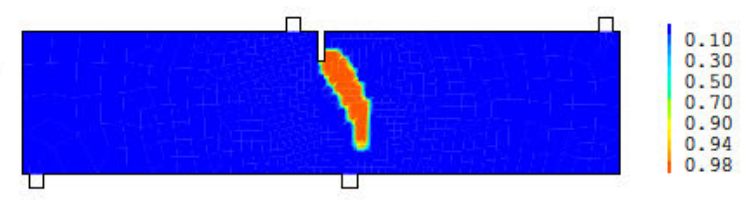

(b)

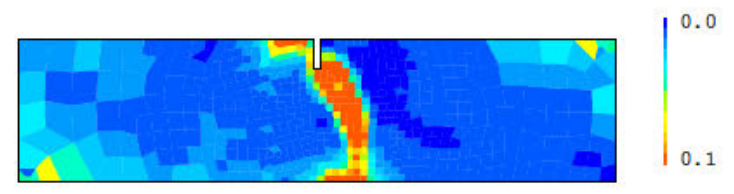

(d)

Figure 17. SENB test. Initial approximation: (a) Mesh 0: 659 elem., 719 nodes; (b) damage; (c) deformed mesh $(\times 300)$; (d) error field. Global relative error: $3.96 \%$

above a threshold set a priori of $2 \%$, so adaptivity is required.

The error field of Fig. 17(d) is translated into the mesh of Fig. 18(a). Note the element concentration in the crack and the central supports. This finer mesh leads to a better definition of the damaged zone, see Fig. 18(b). The error estimator now detects that the largest errors are associated to the edges of the cracked zone, see Fig. 18(d). The global relative error of $2.11 \%$ is still slightly above the error goal, so another adaptive iteration is performed. The outcome of this second iteration is shown in Fig. 19. The qualitative results of iteration 1 are confirmed: (1) small elements are needed to control the error in the damaged zones and close to the loading platens and (2) error is larger in the edges than in the centre of the crack. The global relative error of $1.77 \%$ is below the threshold of $2 \%$, so the adaptive iterative process stops.

The structural response is shown in Fig. 20, where the total load is plotted versus the CMSD. The peak load of around $60 \mathrm{kN}$ (with mesh 2) and the post-peak softening are in good agreement with the experiments [29]. Note that there is a significant quantitative difference between the solutions with meshes 0 and 2 . The result with the initial mesh clearly overestimates the peak 


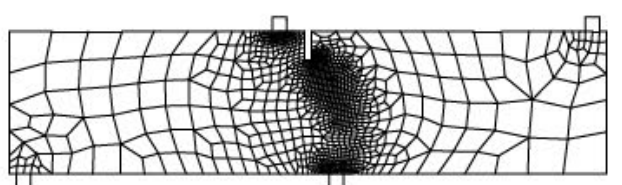

(a)

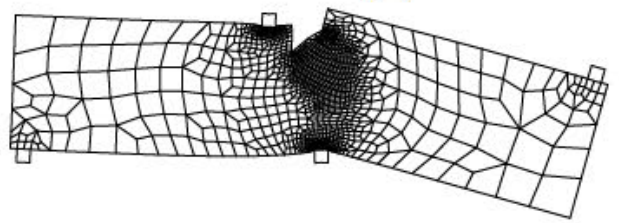

(c)

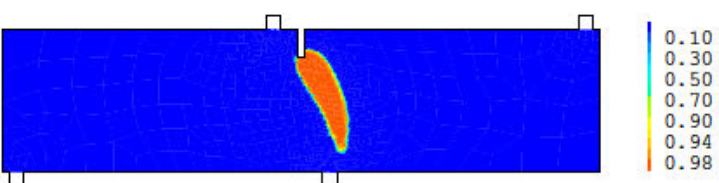

(b)

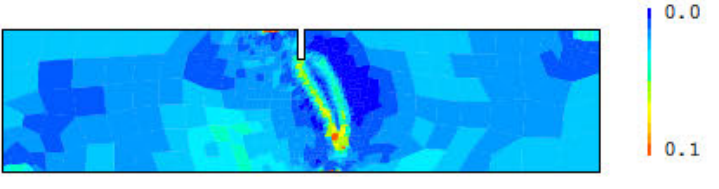

(d)

Figure 18. SENB test. Iteration 1: (a) Mesh 1: 1155 elem., 1228 nodes; (b) damage; (c) deformed mesh $(\times 300)$; (d) error field. Global relative error: $2.11 \%$

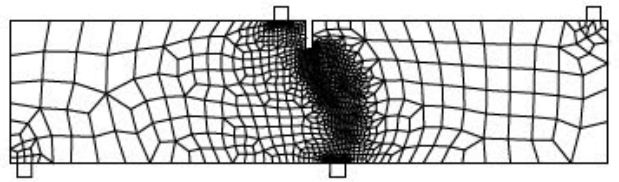

(a)

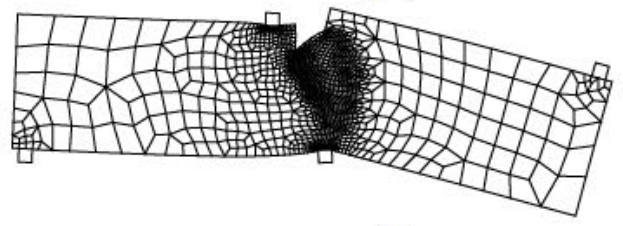

(c)

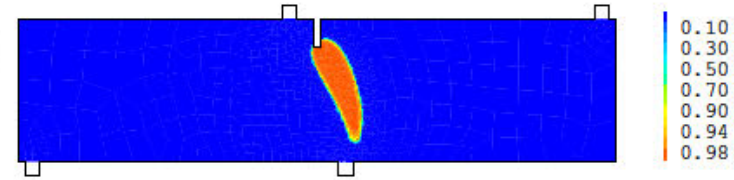

(b)

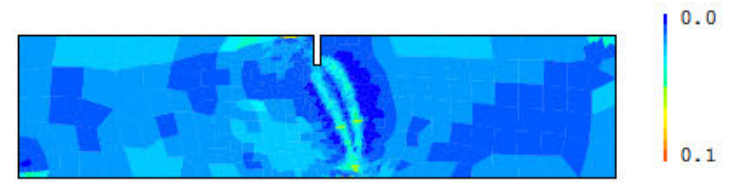

(d)

Figure 19. SENB test. Iteration 2: (a) Mesh 2: 1389 elem., 1469 nodes; (b) damage; (c) deformed mesh $(\times 300)$; (d) error field. Global relative error: $1.77 \%$

load. The adaptive strategy based on error estimation enables an accurate prediction of the structural response.

\section{Concluding Remarks}

Efficiency and reliability are two crucial issues in any field of computational mechanics. We have addressed them here in the context of failure analysis of quasi-brittle materials by means of nonlocal damage models. The following conclusions may be pointed out:

- An adaptive strategy driven by an error estimator is required to ensure the quality of the numerical results in an objective manner.

- The error estimator may be based on local computations - a common approach in residual-type error estimators -, provided that the damage model is slightly modified. The basic idea is that the error in the local state vari- 


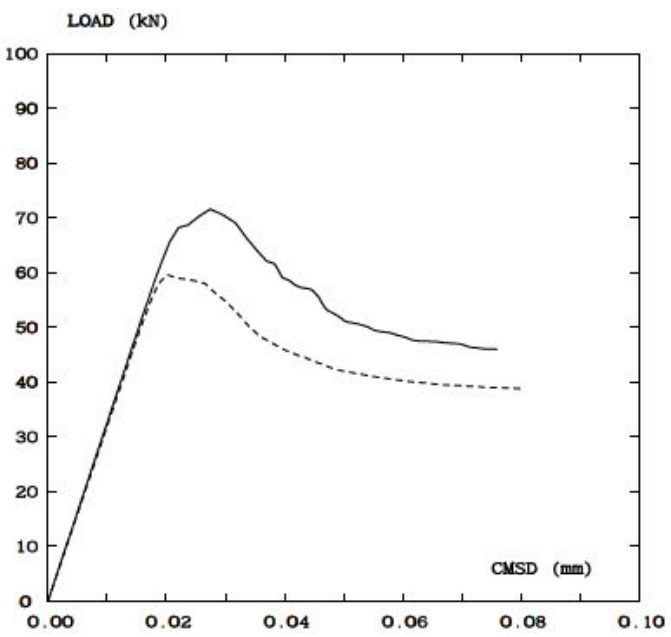

Figure 20. SENB test. Total load versus crack-mouth sliding displacement (CMSD) for meshes 0 (solid line) and 2 (dashed line)

able, rather than the variable itself, is averaged.

- Advanced control strategies are needed to account in an efficient manner for the highly nonlinear and localized structural response.

- As expected, the consistent tangent matrix provides quadratic convergence in Newton equilibrium iterations. If the extra fill-in of this matrix caused by nonlocality is a critical factor, it can be avoided with an inner iterative loop.

- With computational efficiency in mind, we have presented and briefly discussed a new nonlocal damage model based on the nonlocal average of displacements (rather than the state variable). Our preliminary results indicate that this model is sound from a physical point of view - regarding its regularisation capabilities - and has very attractive numerical properties, especially regarding the computation of the consistent tangent matrix.

\section{A Nonlocal Averaging of Displacements with Consistency of Order 1}

As discussed in Sect. 3, consistency of order 1 is needed for the nonlocal average of displacements. This can be achieved by means of a moving leastsquares fitting, a standard technique in particle (or meshless) methods [30,31], briefly summarized here.

For a given point $\mathrm{x}$, the goal is to approximate a function $f$ in the neighbourhood $V_{x}$ (in our case, associated to a characteristic length) of $\mathrm{x}$ by means of a polynomial of degree $n, \mathbf{f}_{l_{c}}(\mathbf{z})$,

$$
f_{l_{\mathrm{c}}}(\mathbf{z})=\mathbf{P}^{T}(\mathbf{z}) \mathbf{c}(\mathbf{x})
$$


where $\mathbf{P}(\mathbf{z})=\left\{p_{0}(\mathbf{z}), p_{1}(\mathbf{z}), \ldots, p_{n}(\mathbf{z})\right\}^{T}$ contains a complete basis of the space of polynomials of degree less or equal to $n$. The vector of coefficients $\mathbf{c}(\mathbf{x})$ is obtained by a least squares fitting with the local scalar product

$$
\langle g, h\rangle_{x}=\int_{V_{x}} \alpha_{0}(\mathbf{x}-\xi) g(\xi) h(\xi) \mathrm{d} \xi,
$$

centered at $\mathbf{x}$ and weighted with $\alpha_{0}$. The resulting normal equations are

$$
\mathbf{M}(\mathbf{x}) \mathbf{c}(\mathbf{x})=\mathbf{b}(\mathbf{x})
$$

with

$$
\mathbf{M}(\mathbf{x})=\int_{V_{x}} \alpha_{0}(\mathbf{x}-\boldsymbol{\xi}) \mathbf{P}(\boldsymbol{\xi}) \mathbf{P}^{T}(\boldsymbol{\xi}) \mathrm{d} \boldsymbol{\xi}
$$

and

$$
\mathbf{b}(\mathbf{x})=\int_{V_{x}} \alpha_{0}(\mathbf{x}-\xi) \mathbf{P}(\xi) f(\boldsymbol{\xi}) \mathrm{d} \xi
$$

The smoothed function at $\mathbf{x}, \tilde{f}(\mathbf{x})$, is obtained by evaluating the local polynomial approximation (A.1) at $\mathbf{z}=\mathbf{x}$, that is

$$
\tilde{f}(\mathbf{x})=f_{l_{\mathrm{c}}}(\mathbf{z}=\mathbf{x})=\mathbf{P}^{T}(\mathbf{x}) \mathbf{c}(\mathbf{x}) .
$$

For $n=0$ (consistency of order 0), (A.6) boils down to the standard nonlocal averaging represented by (3) and (9). For $n=1$ (consistency of order 1), (A.3) is a linear system of dimension 2 to be solved at each node. This is done only once, at the beginning of the computation, and coefficients $c_{0}(\mathbf{x})$ and $c_{1}(\mathbf{x})$ are stored and reused throughout the analysis.

\section{References}

[1] J. Lemaitre, J.-L. Chaboche, Mechanics of solid materials, Cambridge University Press, Cambridge, 1990.

[2] R. de Borst, J. Pamin, R. Peerlings, L. Sluys, On gradient-enhanced damage and plasticity models for failure in quasi-brittle and frictional materials, Comput. Mech. 17 (1-2) (1995) 130-141.

[3] G. Pijaudier-Cabot, Z. Bažant, Nonlocal damage theory, J. Eng. Mech.-ASCE 118 (10) (1987) 1512-1533.

[4] Z. Bažant, G. Pijaudier-Cabot, Nonlocal continuum damage, localization instability and convergence, J. Appl. Mech.-Trans. ASME 55 (2) (1988) 287293.

[5] J. Mazars, G. Pijaudier-Cabot, Continuum damage theory - application to concrete, J. Eng. Mech.-ASCE 115 (2) (1989) 345-365. 
[6] A. Rodríguez-Ferran, I. Arbós, A. Huerta, Adaptive analysis based on error estimation for nonlocal damage models, Revue européene des éléments finis $10(2-4)(2001)$ 193-207.

[7] A. Rodríguez-Ferran, A. Huerta, Error estimation and adaptivity for nonlocal damage models, Int. J. Solids Struct. 37 (48-50) (2000) 7501-7528.

[8] M. Jirásek, B. Patzák, Consistent tangent stiffness for nonlocal damage models, Comput. Struct. 80 (14-15) (2002) 1279-1293.

[9] M. Jirásek, Nonlocal models for damage and fracture: comparison of approaches, Int. J. Solids Struct. 35 (31-32) (1998) 4133-4145.

[10] Z. Bažant, M. Jirásek, Nonlocal integral formulations of plasticity and damage: survey of progress, J. Eng. Mech.-ASCE 128 (11) (2002) 1119-1149.

[11] A. Huerta, G. Pijaudier-Cabot, Discretization influence on regularization by two localization limiters, J. Eng. Mech.-ASCE 120 (6) (1994) 1198-1218.

[12] J. Mazars, A description of micro- and macroscale damage of concrete structures, Eng. Fract. Mech. 25 (5-6) (1986) 729-737.

[13] J. de Vree, W. Brekelmans, M. van Gils, Comparison of nonlocal approaches in continuum damage mechanics, Comput. Struct. 55 (4) (1995) 581-588.

[14] J. Mazars, G. Pijaudier-Cabot, C. Saouridis, Size effect and continuous damage in cementitious materials, Int. J. Fract. 51 (2) (1991) 159-173.

[15] P. Pegon, A. Anthoine, Numerical strategies for solving continuum damage problems involving softening: application to the homogenization of masonry, in: Proceedings of the Second International Conference on Computational Structures Technology, Athens, 1994.

[16] H. Askes, L. Sluys, Remeshing strategies for adaptive ALE analysis of strain localisation, Eur. J. Mech. A-Solids 19 (3) (2000) 447-467.

[17] G. Pijaudier-Cabot, A. Huerta, Finite element analysis of bifurcation in nonlocal strain softening solids, Comput. Methods Appl. Mech. Eng. 90 (1-3) (1991) 905-919.

[18] A. Simone, H. Askes, R. H. J. Peerlings, L. J. Sluys, Interpolation requirements for implicit gradient-enhanced continuum damage models, Commun. Numer. Methods Eng. 19 (7) (2003) 563-572.

[19] M. A. Crisfield, Non-linear finite element analysis of solids and structures. Volume 1: Essentials, John Wiley \& Sons, Chichester, 1991.

[20] T. Belytschko, W. Liu, B. Moran, Nonlinear finite elements for continua and structures, John Wiley \& Sons, Chichester, 2000.

[21] C. Kelley, Iterative methods for linear and nonlinear equations, Vol. 16 of Frontiers in Applied Mathematics, Society for Industrial and Applied Mathematics, Philadelphia, 1995. 
[22] C. L. Bellégo, Couplages chimie-mécanique dans les structures en béton attaquées per l'eau: étude expérimentale et analyse numérique, Ph.D. thesis, E.N.S. Cachan (February 2001).

[23] A. Huerta, P. Díez, Error estimation including pollution assessment for nonlinear finite element analysis, Comput. Methods Appl. Mech. Eng. 181 (1-3) (2000) 21-41.

[24] J. Sarrate, A. Huerta, Efficient unstructured quadrilateral mesh generation, Int. J. Numer. Methods Eng. 49 (10) (2000) 1327-1350.

[25] P. Díez, J. Egozcue, A. Huerta, A posteriori error estimation for standard finite element analysis, Comput. Methods Appl. Mech. Eng. 163 (1-4) (1998) 141157.

[26] P. Díez, M. Arroyo, A. Huerta, Adaptivity based on error estimation for viscoplastic softening materials, Mech. Cohesive-Frict. Mater. 5 (2) (2000) 87112.

[27] P. Díez, A. Huerta, Error estimation for adaptivity in assumed-strain models for linear and nonlinear shells, Comput. Mech. Submitted.

[28] A. Rodríguez-Ferran, A. Huerta, Adapting broyden method to handle linear constraints imposed via lagrange multipliers, Int. J. Numer. Methods Eng. 46 (12) (1999) 2011-2026.

[29] A. Carpinteri, S. Valente, G. Ferrara, G. Melchiorri, Is mode II fracture energy a real material property?, Comput. Struct. 48 (3) (1993) 397-413.

[30] P. Lancaster, K. Salkauskas, Surfaces generated by moving least squares methods, Math. Comput. 37 (155) (1981) 141-158.

[31] A. Huerta, S. Fernández-Méndez, Locking in the incompressible limit for the Element Free Galerkin method, Int. J. Numer. Methods Eng. 51 (11) (2001) $1361-1383$. 